\title{
ON THE BINARY HELIUM STAR DY CENTAURI: CHEMICAL COMPOSITION AND EVOLUTIONARY STATE
}

\author{
Gajendra Pandey ${ }^{1}$, N. Kameswara RaO ${ }^{1}$, C. Simon JefFery ${ }^{2}$, and David L. Lambert ${ }^{3}$ \\ ${ }^{1}$ Indian Institute of Astrophysics, Bangalore 560034, India; pandey@iiap.res.in, nkrao@iiap.res.in \\ ${ }^{2}$ Armagh Observatory, Collage Hill, Armagh BT61 9DG, UK; csj@arm.ac.uk \\ ${ }^{3}$ The W. J. McDonald Observatory and Department of Astronomy, University of Texas at Austin, Austin, TX 78712-1083, USA; dl1@astro.as.utexas.edu \\ Received 2014 June 26; accepted 2014 July 28; published 2014 September 9
}

\begin{abstract}
DY Cen has shown a steady fading of its visual light by about one magnitude in the last $40 \mathrm{yr}$, suggesting a secular increase in its effective temperature. We have conducted non-local thermodynamic equilibrium (LTE) and LTE abundance analyses to determine the star's effective temperature, surface gravity, and chemical composition using high-resolution spectra obtained over two decades. The derived stellar parameters for three epochs suggest that DY Cen has evolved at a constant luminosity and has become hotter by about $5000 \mathrm{~K}$ in $23 \mathrm{yr}$. We show that the derived abundances remain unchanged for the three epochs. The derived abundances of the key elements, including $\mathrm{F}$ and $\mathrm{Ne}$, are as observed for the extreme helium stars resulting from a merger of a He white dwarf with a $\mathrm{C}-\mathrm{O}$ white dwarf. Thus DY Cen by chemical composition appears to also be a product of a merger of two white dwarfs. This appearance seems to be at odds with the recent suggestion that DY Cen is a single-lined spectroscopic binary.
\end{abstract}

Key words: stars: abundances - stars: atmospheres - stars: chemically peculiar - stars: evolution - stars: fundamental parameters - stars: individual (DY Cen)

\section{INTRODUCTION}

The hydrogen-deficient giant DY Centauri is commonly known as an R Coronae Borealis (RCB) variable (Hoffleit 1930), although no RCB-type activity has been observed since that reported in 1930. From its color, it was known to be significantly hotter than most other RCB stars (Kilkenny \& Whittet 1984), while a high-resolution spectrum obtained in 1987 showed it to have an effective temperature and surface composition similar to that of the hotter extreme helium (EHe) stars (Jeffery \& Heber 1993) (JH93), albeit with an apparently low iron abundance and a high $(10 \%)$ hydrogen abundance. An identification of $\mathrm{RCB}$ and EHe stars with stars evolving on a post-white-dwarf merger track has become increasingly strong in recent years (Saio \& Jeffery 2002; Pandey et al. 2006; Clayton et al. 2007; Jeffery et al. 2011); the assumption was that DY Cen also lies on this track.

Recent evidence suggests that this may not be so. The conclusion that DY Cen represents a substantially different type of star to the RCB and EHe stars was hinted at by De Marco et al. (2002), who highlighted a number of discrepant radial velocity measurements and demonstrated a systematic increase in $V$ magnitude during an interval of some $80 \mathrm{yr}$. Rao et al. (2012) demonstrated that DY Cen is a single-lined spectroscopic binary with an orbital period of $39 \mathrm{~d}$; all other EHe and RCB stars are single stars, as expected for a white-dwarf-merger origin. DY Cen and another "RCB" star with significant hydrogen, V854 Cen, show other differences to normal RCB stars. Spitzer Space Telescope observations show $\mathrm{C}_{60}$ in both DY Cen and V854 Cen but not in other RCB stars (García-Hernández et al. 2011). DY Cen's spectrum is also unusual in that nebular emission lines are present at maximum light. Note that there are three other "hot" RCB stars with emission lines: MV Sgr, V348 Sgr, and HV 2671. DY Cen and MV Sgr seem to have RCB abundances, while V348 Sgr and HV 2671 do not (see Clayton et al. (2011), and references therein). The present paper was stimulated in part by the systematic and secular fading of DY Cen from a visual magnitude of 12.2 in 1970 to nearer
13.2 in 2010. To explain this requires either a steady increase in extinction, a fall in intrinsic brightness, a change in effective temperature (which changes the bolometric correction), or a combination of all three. Given the magnitude and rapidity of the change, any one of these has profound consequences for interpreting the evolutionary status of DY Cen.

This paper first examines the implications of the visualmagnitude variation assuming evolution at constant luminosity. It then carries out a fine analysis for effective temperature, surface gravity, and chemical composition using high-quality spectra obtained over two decades. Judged by chemical composition, especially by the F and Ne abundances, DY Cen closely resembles hot EHes. Since EHes are generally considered to be single stars and formed by the merger of a Helium white dwarf with a $\mathrm{C}-\mathrm{O}$ white dwarf, identification of DY Cen with the EHes challenges the recent identification of DY Cen as a single-lined spectroscopic binary.

\section{SECULAR FADING OF DY CEN}

Drilling (1986) noted that if the EHe and RCB stars share the same luminosity, they show a steady fading of the absolute visual magnitude toward a higher effective temperature $\left(T_{\text {eff }}\right)$. The photometric variation of DY Cen with time was summarized by De Marco et al. (2002) and Rao et al. (2013), showing a fading in visual magnitude by about 1.3 magnitudes over the previous century with dramatic change in the nebular emission line fluxes, prompting the suggestion that it could be associated with a secular increase in effective temperature on a timescale of decades.

In order to calibrate the star's fading, we have taken the theoretical spectral energy distributions (SEDs) for a grid of line-blanketed hydrogen-deficient model atmospheres (Behara \& Jeffery 2006), computed with a chemical composition appropriate to that of DY Cen. Since the SED total flux is proportional to the fourth power of $T_{\text {eff }}$, each SED in the model grid was divided by this quantity in order to apply a constant luminosity approximation. Each SED was then convolved with the $B$ - and 


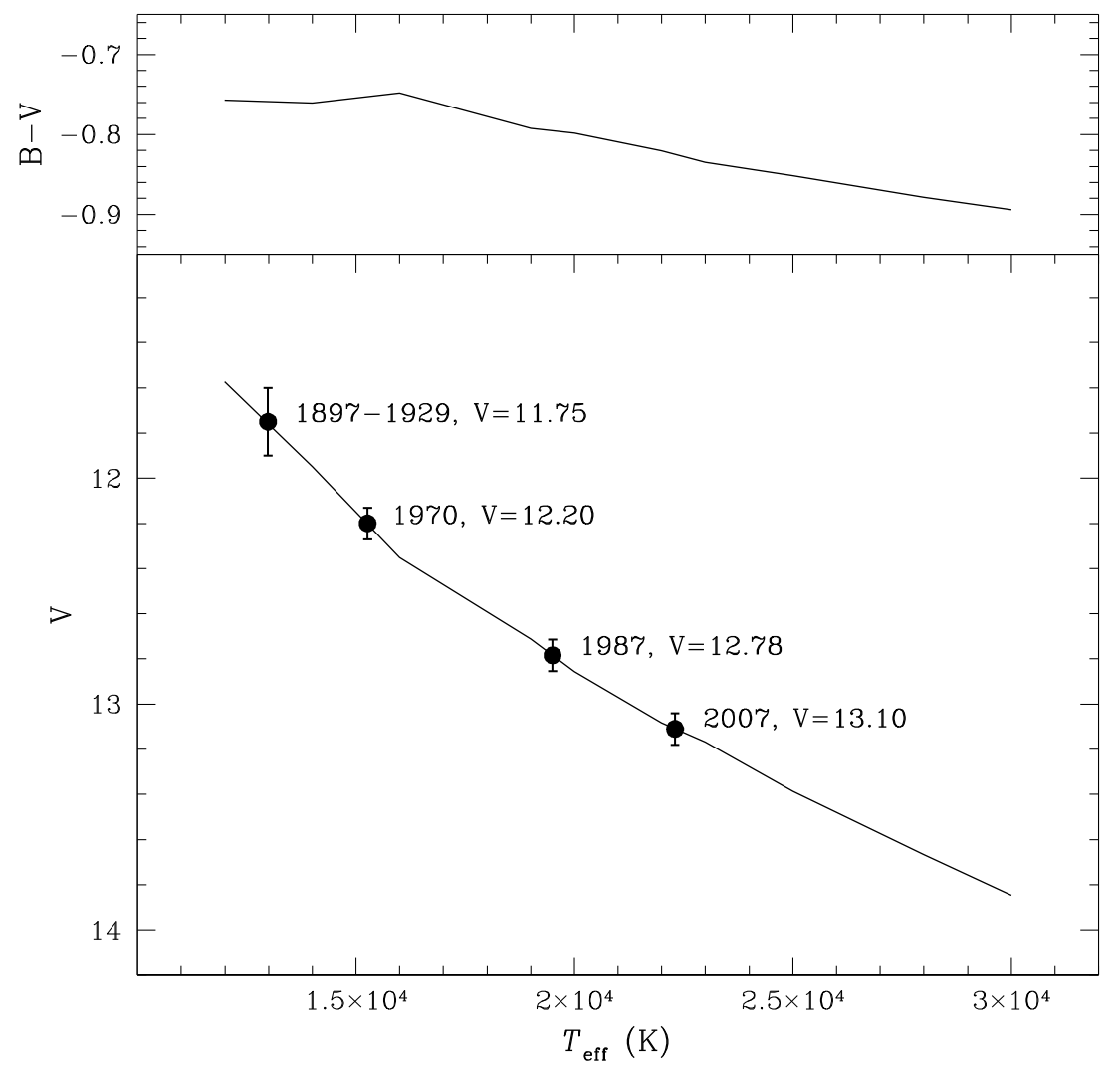

Figure 1. Lower panel: theoretical zero-point-corrected $V$ magnitudes vs. $T_{\text {eff }}$. Observed $V$ magnitudes are shown by filled circles with errors and epoch. The effective temperatures for the 1987 point are taken from spectroscopy. Upper panel: theoretical $B-V$ colors vs. $T_{\text {eff }}$.

$V$-band-filter response functions given in Table 2 of Bessell (1990), integrated to obtain the total flux in the $V$ band, and converted to a magnitude.

An arbitrary zero point is obtained by comparing the theoretical $V$ magnitude corresponding to $T_{\text {eff }}=19,500 \mathrm{~K}$ in 1987 May measured spectroscopically by JH93 with a mean value of $V=12.784$ measured by Pollacco \& Hill (1991) in 1987 May and June. The zero-point-corrected theoretical $V$ magnitudes and the $B-V$ colors are shown as a function of $T_{\text {eff }}$ in Figure 1, together with observed $V$ magnitudes from 1930, 1970, 1987 (Hoffleit 1930; Marino \& Walker 1971; Pollacco \& Hill 1991), and 2007 (AAVSO ${ }^{4}$ ). Hoffleit's photographic magnitudes are converted to $V$ magnitude by a recipe given by De Marco et al. (2002).

Figure 1 suggests that if the luminosity has remained constant, an explanation for the secular fading of DY Cen can be provided by an increase in effective temperature from about $14,000 \mathrm{~K}$ in 1930 to about $25,000 \mathrm{~K}$ in 2010 . It remains to be shown that this is the only explanation which can account for the observations.

In the following sections, high-resolution optical spectra from 1987 to 2010 are analyzed to determine if the star has evolved at constant luminosity with concomitant variations in effective temperature and surface gravity. In addition, the chemical composition of DY Cen is determined anew using the superior spectra now available.

\section{OBSERVATIONS}

High-resolution spectra of DY Cen are available from the nights of 1987 April 17, 2002 June 24, and several nights

\footnotetext{
4 http://www.aavso.org
}

between 2010 February 27 and 2010 March 2. All spectra were obtained at maximum light, there having been no minima recorded over the period of these observations, according to visual estimates from AAVSO. The data were reduced using standard procedures appropriate to the instrument, as described by JH93 and Rao et al. (2012).

The 1987 spectrum was obtained with CASPEC, the Cassegrain échelle spectrograph on the $3.6 \mathrm{~m}$ telescope of the European Southern Observatory at La Silla, Chile. The spectrum covers the spectral range $4000-4900 \AA$ at a (two-pixel) resolution of about 22,000 . The spectrum was analyzed in detail by JH93.

The 2002 spectrum was obtained with UCLES, the University College échelle spectrograph on the $3.9 \mathrm{~m}$ Anglo-Australian Telescope at Siding Spring Observatory, Australia. The spectrum covers the spectral range $3800-5100 \AA$ at a resolution of 100,000 with a signal-to-noise ratio $(\mathrm{S} / \mathrm{N})$ of 70 , but when smoothed to the resolution of the 2010 UVES spectra the UCLES spectrum has a $\mathrm{S} / \mathrm{N}$ comparable to that of the former. Further details were given by Rao et al. (2012).

Four spectra were acquired in 2010 February and March with UVES, the cross-dispersed échelle spectrograph (Dekker et al. 2000) on the Very Large Telescope of the European Southern Observatory at Cerro Paranal, Chile. A resolution $R \equiv \lambda / \Delta \lambda \approx 34,000$ was estimated from the telluric lines in the $5920 \AA$ region. The available spectra cover the wavelength regions 3300-4500 $\AA$ and 5700-7500 $\mathrm{A}$. In the blue spectrum, the $\mathrm{S} / \mathrm{N}$ exceeds 200 at the above wavelengths. The spectrum from 2010 February 27 was chosen for an abundance analysis because the lines were least affected by emission in the core, and the absorption line profiles are the most symmetric. Emission cores are prominent in hydrogen Balmer lines, He I lines, and 
$\mathrm{C}$ II lines. The emission appears to be variable on a timescale of weeks, since the 2010 February 27 spectrum shows little or no core emission compared with the 2010 March 2 spectrum. Several nebular lines, including [O I], [O II], [N II], and [S II], are also present (Rao et al. 2013).

Spectra at all three epochs are dominated by photospheric absorption. The vast majority of lines arise from H I, He I, C II, C III, N II, N III, O II, O III, Ne I, and Ne II. A few lines from other ions are present, but care must be taken to account for blending of these lines with lines from the above species which dominate the spectrum. Lines were identified using the Revised Multiplet Table (RMT; Moore 1972), tables of spectra of H, C, N, and O (Moore 1993), and the NIST Atomic Spectra Database. ${ }^{5}$

The primary information required from these spectra are the effective temperature and surface gravity and the chemical composition of DY Cen at each epoch. Quantitative analysis is applied consistently to each spectrum.

\section{QUANTITATIVE FINE ANALYSES}

Determination of the atmospheric parameters and the chemical composition is based on line-blanketed model atmospheres. The effective temperature $T_{\text {eff }}$ and surface gravity $g$ are obtained from the intersection of loci in the $T_{\text {eff }}$ versus $\log g$ plane. These loci include several expressing ionization equilibrium (e.g., C II/C III and O II/O III) and others derived from fits to the Stark-broadened profiles of the He I lines, as illustrated below. The microturbulent velocity $\xi$ is determined from the $\mathrm{O}$ II lines spanning a range in equivalent width.

In principle, determination of chemical composition is an iterative process which concludes when the composition adopted in the computation of the model atmosphere equals the composition derived from a spectrum. Test calculations with models computed for $\mathrm{C} / \mathrm{He}$ of $0.3 \%-1 \%$ and $\mathrm{H} / \mathrm{He}$ of 0.0001 and 0.1 give essentially the same atmospheric structure and, thence, the same atmospheric parameters, including the composition.

For DY Cen, photoionization of neutral helium is the principal source of continuous opacity at optical wavelengths. Thus lines of another species, e.g., $\mathrm{C}$ II, are sensitive to the $\mathrm{C} / \mathrm{He}$ ratio. The abundances are given as $\log \epsilon(\mathrm{X})$ and normalized with respect to $\log \Sigma \mu_{\mathrm{X}} \epsilon(\mathrm{X})=12.15$, where $\mu_{\mathrm{X}}$ is the atomic weight of element $\mathrm{X}$. With bound-free He transitions providing the opacity, the strength of the He I lines is not very temperature-sensitive; the excitation potential of the continuous opacity-providing levels is but slightly elevated with respect to the levels providing the observed lines. A similar consideration applies to many of the lines of other ions which have high excitation potentials but are similar to those of He providing continuous opacity.

Under DY Cen's atmospheric conditions, the approximation of local thermodynamic equilibrium (LTE) is expected to fail for some species. In recognition of this failure, the abundance analyses were carried out for non-LTE model atmospheres and for non-LTE (and LTE) line formation for all major elements and some minor elements. Partially blanketed nonLTE model atmospheres were computed with the code TLUSTY (Hubeny 1988; Hubeny \& Lanz 1995) using atomic data and model atoms provided on the TLUSTY home page. ${ }^{6}$ The microturbulence of $\xi=10 \mathrm{~km} \mathrm{~s}^{-1}$ was used for computing the model atmospheres. These model atmospheres included both bound-free and bound-bound transitions of $\mathrm{H}, \mathrm{He}, \mathrm{C}, \mathrm{N}, \mathrm{O}$, and $\mathrm{Ne}$ in NLTE. The adopted model atoms, with their number of

\footnotetext{
5 http://www.nist.gov/pml/data/asd.cfm

6 http://nova.astro.umd.edu/index.html
}

levels given in brackets, are $\mathrm{HI}(9), \mathrm{He} \mathrm{I}(14), \mathrm{He} \mathrm{II}(14), \mathrm{CI}(8)$, C II(11), C III(12), C IV(13), N I(13), N II(6), N III(11), N IV(12), O I(22), O II(29), O III(29), Ne I(35), Ne II(32), and Ne III(34). Other atoms were considered with lines analyzed in LTE except for the following, for which TLUSTY provides model atoms with the number of levels in brackets: MgII(14), Si II(16), Si III(12), Si IV(13), S II(14), S III(20), Fe II(36), and Fe III(50).

Model atmospheres in LTE were computed using TLUSTY and were also taken from an extensive grid of LTE models described by Behara \& Jeffery (2006). The latter models were constructed using the fully line-blanketed LTE code STERNE (Behara \& Jeffery 2006), which incorporates Opacity Project bound-free opacities for all important elements up to and including iron and bound-bound atomic transitions corresponding to some $10^{6}$ lines by means of an opacity-sampling (OS) formalism (Behara \& Jeffery 2006). As a starting approximation, a composition corresponding to that of JH93 was adopted, with the exception that the iron abundance was scaled to be solar relative to the silicon abundance. An important feature of the OS approach is that the microturbulent velocity $\xi$ can easily be adjusted to be consistent with that measured from the observed spectrum. A large value of $\xi$ was found by JH93 and confirmed by the present analyses (see below). A model grid was computed covering the ranges $T_{\text {eff }}=16,000(1000) 30,000 \mathrm{~K}$ and $\log g=1.7(0.1) 3.0 \mathrm{cgs}$. Test calculations showed that LTE TLUSTY and LTE STERNE models for parameters applicable to DY Cen give essentially the same atmospheric parameters and elemental abundances. It is thus very likely that the nonLTE effects estimated from comparison of results for LTE and non-LTE TLUSTY atmospheres are representative of those that would be found from STERNE models were the latter available in a non-LTE variety.

An extensive LTE analysis of the blue optical spectrum of DY Cen was reported by JH93. Ionization equilibrium was used to establish the effective temperature $T_{\text {eff }}$, Stark-broadened neutral helium line profiles provided the surface gravity $g$, and abundances were obtained for 13 elements from $\mathrm{H}$ to $\mathrm{Fe}$. In addition, the microturbulent velocity $\xi$ and the projected rotation velocity $v_{\text {rot }} \sin i$ were measured from the OII lines. The approximation of LTE for the atomic-level populations was adopted throughout.

In the interim it has been shown that departures from LTE strongly affect the profiles, and especially the cores, of neutral helium lines in EHe stars of similar $T_{\text {eff }}$ to DY Cen (Przybilla et al. 2005), and the lines of $\mathrm{C}, \mathrm{N}$, and $\mathrm{O}$ used to establish atmospheric parameters may also be affected by departures from LTE. Additionally, a complete treatment of line-blanketing is important for the temperature stratification and, hence, the measurement of $T_{\text {eff }}$ of hot He stars (Behara \& Jeffery 2006), but tests show that the line-blanketing adopted for TLUSTY models is a good approximation to complete line-blanketing.

In this paper, we have used TLUSTY and SYNSPEC for calculating LTE and non-LTE model atmospheres and line profiles (Hubeny 1988; Hubeny et al. 1994; Hubeny \& Lanz 1995). Stellar atmospheric parameters are determined from the CASPEC, UCLES, and UVES spectra on the assumption of nonLTE using the H I, He I, C II, C III, C IV, N II, N III, O II, and O III lines. Abundances of elements beyond $\mathrm{O}$ are determined from analyses of lines in the UVES spectra with occasional reference to the CASPEC spectrum and also to the UCLES spectrum, whose $\mathrm{S} / \mathrm{N}$ is comparable to that of a UVES spectrum, when smoothed to the resolving power of a UVES spectrum. Adoption of the UVES spectrum for the "heavy" element analyses is 
warranted by the superior quality of the UVES spectra relative to the CASPEC spectrum. Except where noted, the $g f$-values of the lines are taken from the NIST database. ${ }^{7}$ A few other sources consulted for $g f$-values are referenced in footnotes to the relevant tables.

For the elements $\mathrm{H}$ to $\mathrm{Ne}$, identification of lines suitable for analysis is not a major issue. In particular, ions of $\mathrm{C}$, $\mathrm{N}$, and $\mathrm{O}$ are generally very well represented, and a good selection of clean lines is available. Moore (1993) is the primary source of wavelengths and classifications for these lines. Identifications of the few lines of "heavy" elements are discussed later. Hydrogen Balmer lines are subject to overlying variable emission. Emission swamps the absorption component in early Balmer lines: $\mathrm{H} \delta$ has a strong emission core in the UVES spectra, but strong absorption dominates the line in the CASPEC and UCLES spectra. Strong variable emission is also seen in some $\mathrm{C}$ II lines. Weak emission is the only signature of Balmer lines beyond $\mathrm{H} 17$ at $3697 \AA$ in the UVES spectra. Neutral helium lines are well represented. Early lines in several series exhibit a P Cygni profile, e.g., 5876.6 $\AA$ and $6678.1 \AA$. He I lines are traceable to the short wavelength limit of the UVES spectra. Emission components in $\mathrm{H}$ I, He I, C II, and other lines are likely attributable to the star's extended atmosphere.

\subsection{Non-LTE Analyses}

Line profiles and theoretical equivalent widths were obtained for these model atmospheres using the companion non-LTE code SYNSPEC (Hubeny et al. 1994). Non-LTE abundances were derived by matching the observed absorption profile or its measured equivalent width with the SYNSPEC prediction. Note that features which are unresolved blends of two or more lines were synthesized and matched to the observed feature by adjustment of the abundances.

The procedure for determining the $T_{\text {eff }}, \log g$, and $\xi$ is a standard one. The microturbulent velocity $\xi$ is estimated from the $\mathrm{O}$ II lines because they show a wide range in equivalent width. O II lines with similar lower excitation potentials (LEPs) were used to minimize the temperature dependence: $\mathrm{O}$ II lines were used with LEPs about 23,26 , and $28 \mathrm{eV}$. $\xi$ is found by requiring the abundance to be independent of the measured equivalent width.

For pairs of ions of the same element, insistence upon ionization equilibrium provides a locus in the $\left(T_{\text {eff }}, \log g\right)$ plane. Available potential loci include $\mathrm{C}$ II/C III, C II/C IV, C III/C IV, and $\mathrm{O}$ II/O III. Not all of these loci are available for all spectra.

An additional locus is provided by fits to the strongest, cleanest He I line profiles with their Stark-broadened wings. Predicted line profiles depend on the electron densities and therefore on the temperature and surface gravity.

A final locus is the $T_{\text {eff }}$ from photometry.

The effective temperature and surface gravity are found to be the best overall fit to the intersecting loci.

\subsubsection{CASPEC 1987}

From the 1987 CASPEC spectrum we redetermine the stellar parameters $\left(T_{\mathrm{eff}}, \log g, \xi\right)$ using the non-LTE model atmospheres and the non-LTE line formation code as discussed in the above section. As described earlier, $\xi$ is estimated from the O II lines. The $T_{\text {eff }}$ and $\log g$ are then determined from the He I line profiles, the ionization balance for ( $\mathrm{C}$ II, C III), and the photometric estimate of $T_{\text {eff }}$. For our analysis, we have used the line

\footnotetext{
http://www.nist.gov/pml/data/asd.cfm
}

list given by JH93 with some additions to the $\mathrm{C}$ II and $\mathrm{C}$ III lines. Fits of synthetic spectra convolved with the instrumental profile with a FWHM of $0.2 \AA$ according to JH93 give a projected rotational velocity of $20-25 \mathrm{~km} \mathrm{~s}^{-1}$ from clean O II lines.

Note that for most of the $\mathrm{CNO}$ lines our measured equivalent widths are in good agreement with those of JH93. Hence we have used the JH93 equivalent widths and the most recent $g f$-values (see Section 4). Our non-LTE analysis gives the final model parameters: $\left(T_{\text {eff }}, \log g, \xi\right)=(19,400,2.1,20.0)$, in agreement with the stellar LTE parameters derived by JH93. The CNO lines given in Table 2 are used in this analysis. Observed profiles of the He I $4922 \AA, 4471 \AA$, and $4388 \AA$ line are shown in Figure 2 with predicted non-LTE profiles for non-LTE atmospheres of $T_{\text {eff }}=$ $19,400 \mathrm{~K}$ and two different surface gravities. Predicted profiles include the convolution with the instrumental profile and the projected rotational velocity. At this effective temperature, the surface gravity $\log g \simeq 2.1$ provides a good fit to these He I lines. For the final model, line-by-line non-LTE abundances (including the mean abundance) and the line-to-line scatter are given in Table 2 . The lines giving significantly deviant abundances are marked by a question mark and are not included in estimating the mean. The abundance rms errors due to uncertainty in $T_{\text {eff }}$ and $\log g$ from C II, C III, N II, N III, and $\mathrm{O}$ II are 0.06, 0.24, 0.08, 0.21 , and 0.12 dex, respectively.

\subsubsection{AAT/UCLES 2002}

In our analysis of the AAT/UCLES spectrum, we have adopted the same procedure and nearly the same lines as for the CASPEC spectrum; the spectral bandpasses are almost identical. However, the UCLES spectrum is generally of higher quality, especially if coaddition of pixels is employed to reduce the resolving power to that of the UVES spectrum. The final derived stellar parameters are $\left(T_{\text {eff }}, \log g, \xi\right)=(23,000,2.35$, 23.0). The CNO lines given in Table 2 , and the wings of the observed He I profiles at $4026 \AA$, $4922 \AA$, and $4388 \AA$ (Figure 3 ) are used in this analysis. Synthetic profiles are convolved with the instrumental profile and with the projected rotational profile of $20-27 \mathrm{~km} \mathrm{~s}^{-1}$ determined from a fit to clean $\mathrm{O}$ II lines. The abundance rms errors due to uncertainty in $T_{\text {eff }}$ and $\log g$ from C II, C III, N II, N III, and O II are 0.05, 0.18, 0.08, 0.18, and 0.01 dex, respectively.

\subsubsection{UVES 2010}

Of the UVES spectra, the spectrum with photospheric absorption lines least affected by core emissions was used (2010 February 27). Table 3 of this paper lists the chosen lines of $\mathrm{H}$, $\mathrm{C}, \mathrm{N}$, and $\mathrm{O}$.

A microturbulent velocity $\xi=24 \pm 3 \mathrm{~km} \mathrm{~s}^{-1}$ is obtained from the O II lines. Observed profiles of the He I $4009 \AA$ and $4388 \AA$ line are shown in Figure 4 with predicted non-LTE profiles for a non-LTE atmosphere of $T_{\text {eff }}=25,000 \mathrm{~K}$ and two different surface gravities. The predicted profiles have been convolved with the instrumental profile and the stellar rotation profile. A projected rotational velocity of about $40 \mathrm{~km} \mathrm{~s}^{-1}$ is estimated by using unblended moderately strong lines. The best-fitting theoretical profile $(\log g=2.50)$ provides one point on the $T_{\text {eff }}-\log g$ locus. The chosen lines are those least affected by emission.

To the mean of the loci from the He I profiles we add several loci from application of ionization equilibrium to $\mathrm{C}$ and $\mathrm{O}$ ions and the $T_{\text {eff }}$ from photometry. Figure 5 shows these loci. Their intersection suggests that the best non-LTE model atmosphere has $T_{\text {eff }}=24,800 \pm 600 \mathrm{~K}$ and $\log g=2.50 \pm 0.12$. 


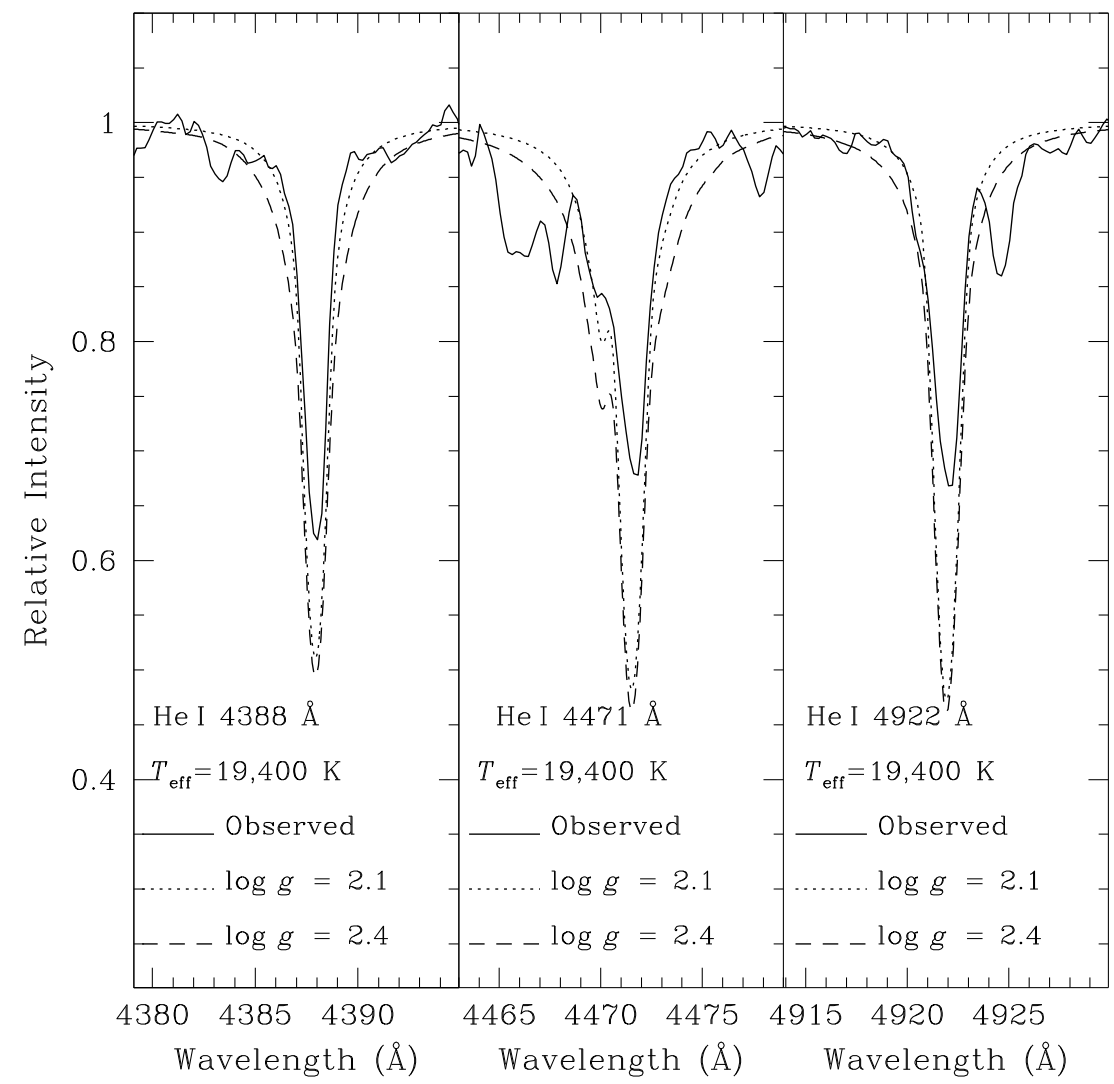

Figure 2. Observed CASPEC spectrum and theoretical NLTE He I line profiles calculated using the NLTE model $T_{\text {eff }}=19,400 \mathrm{~K}$ for two different $\log g$ values. See the key on the figure.

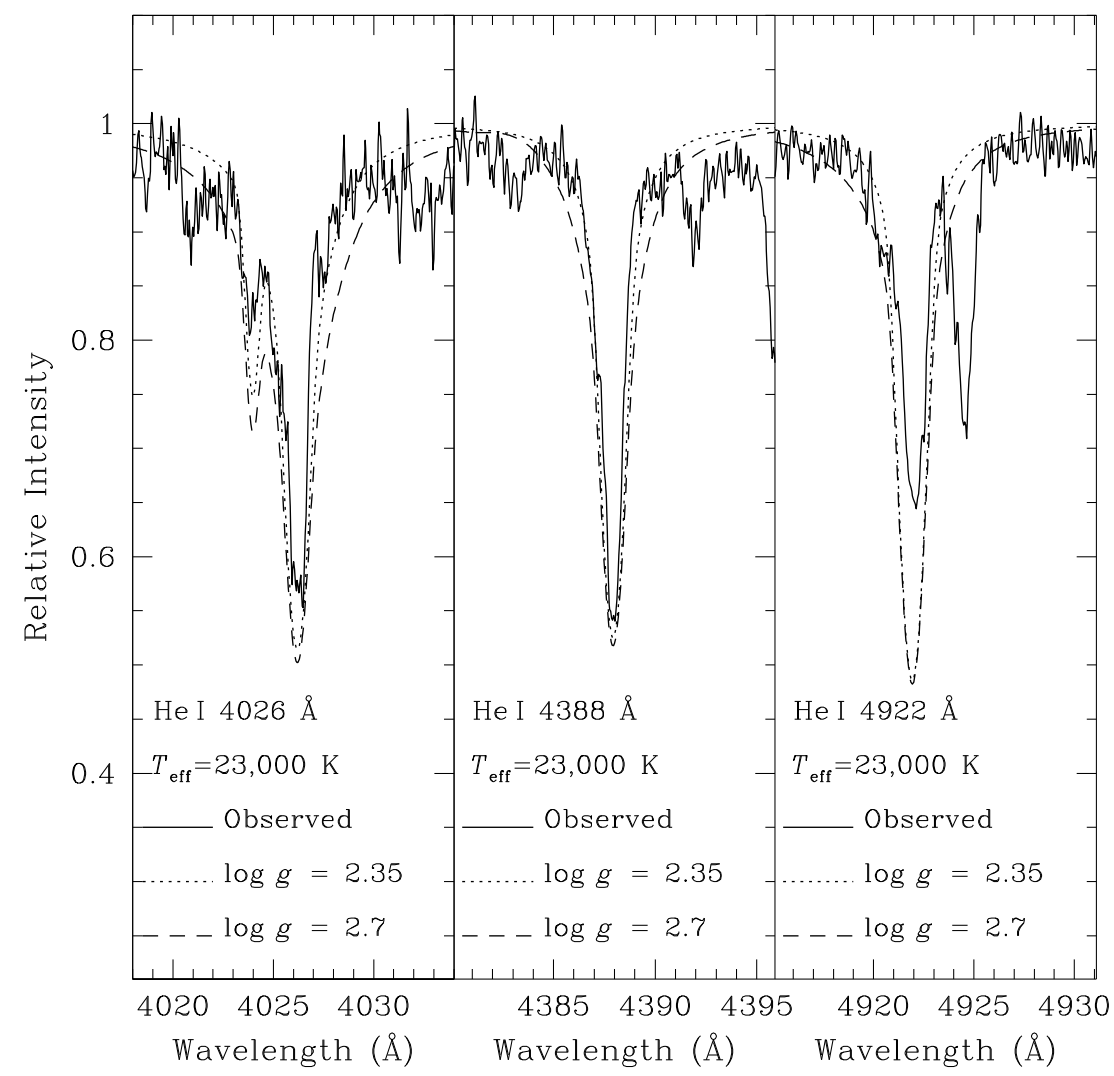

Figure 3. Observed UCLES spectrum and theoretical NLTE He I line profiles calculated using the NLTE model $T_{\text {eff }}=23,000 \mathrm{~K}$ for two different $\log g$ values. See the key on the figure. 


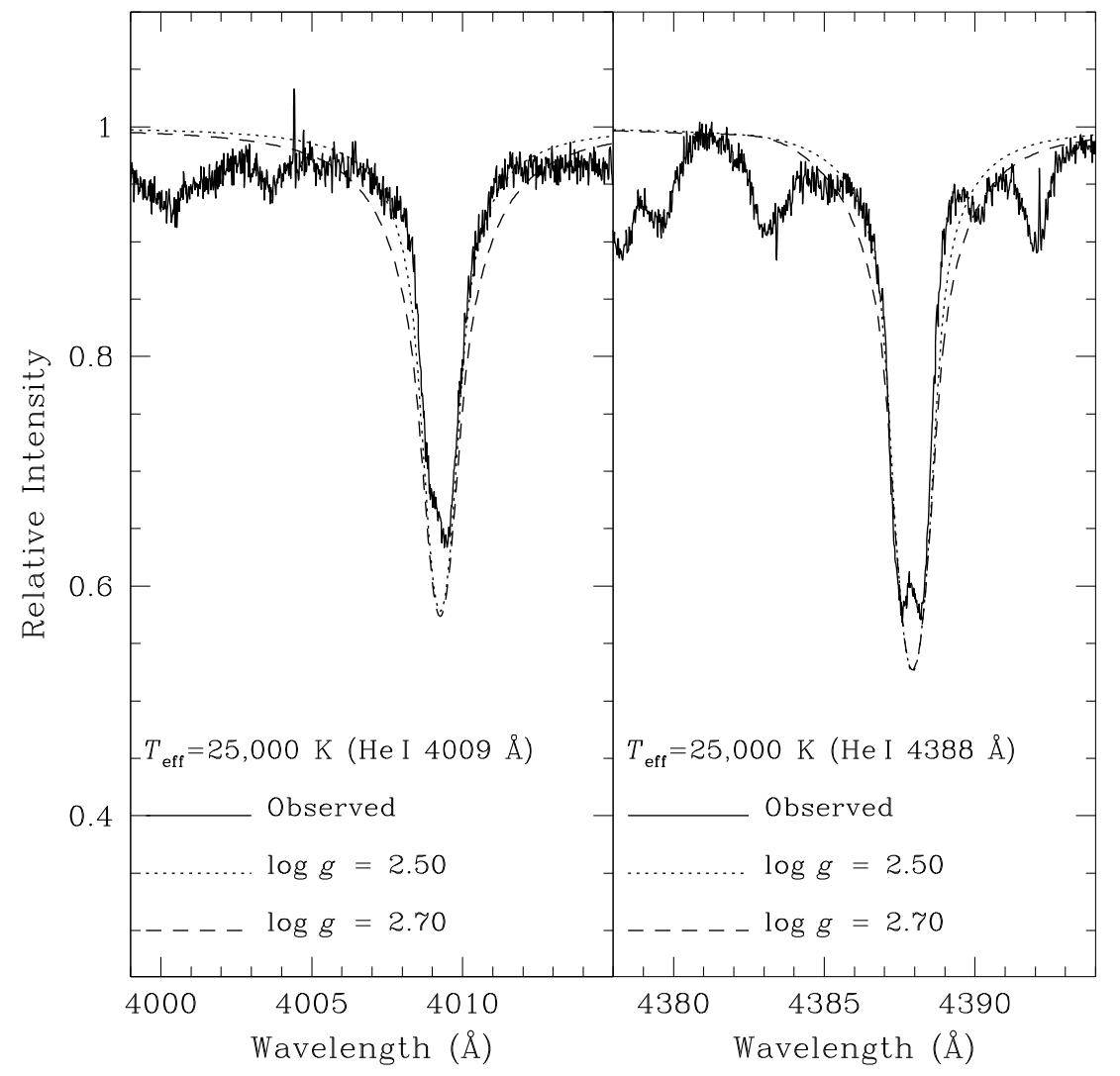

Figure 4. Observed UVES spectrum and theoretical NLTE He I line profiles calculated using the NLTE model $T_{\text {eff }}=25,000 \mathrm{~K}$ for two different $\log g$ values. See the key on the figure.

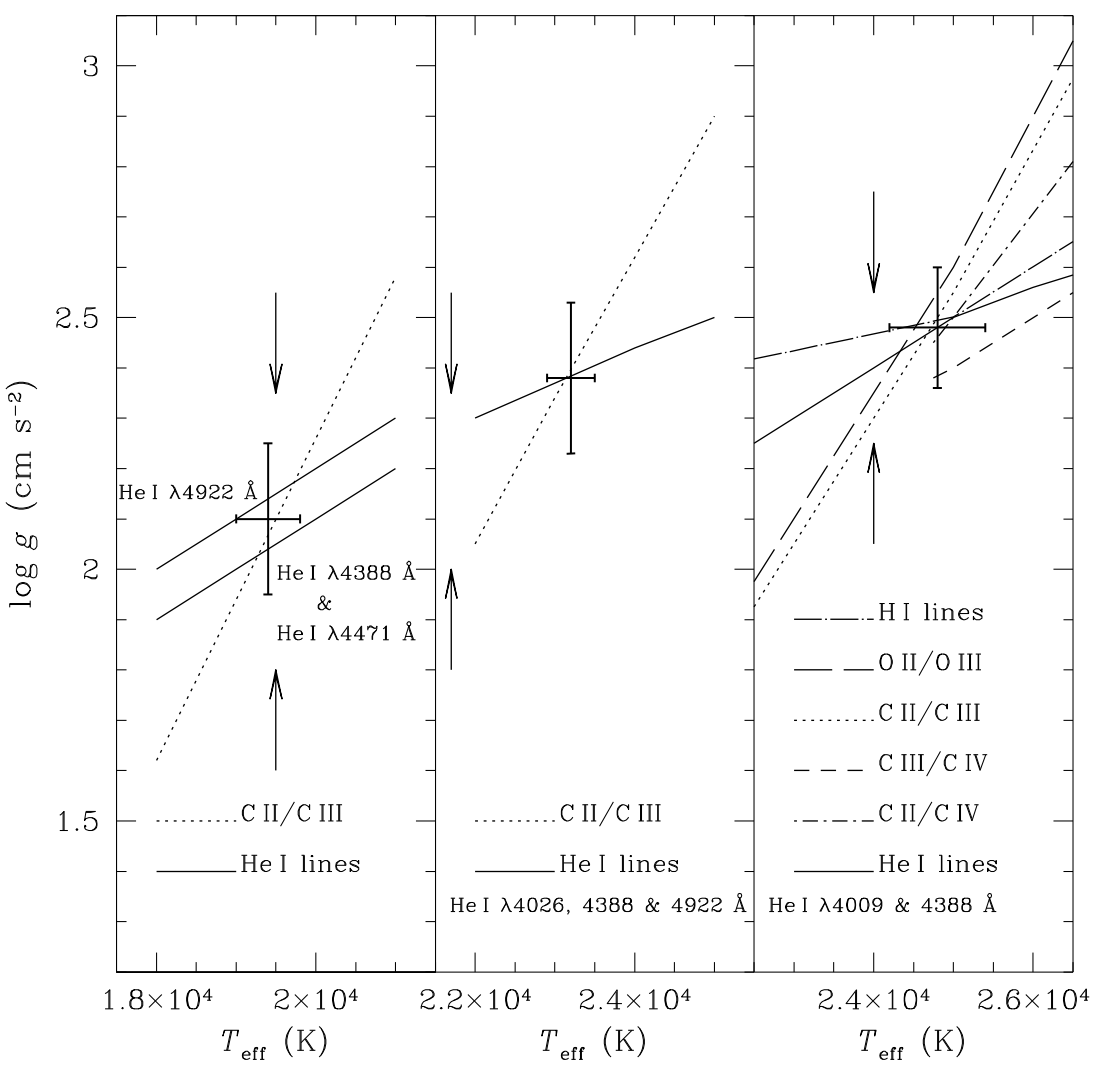

Figure 5. $T_{\text {eff }}$ vs. $\log g$ plane for CASPEC, UCLES, and UVES spectra, from left to right. Loci satisfying ionization equilibria are plotted, see the keys on the figure. The loci satisfying the optical He I line profiles are shown. The locus satisfying the optical H I line profile is also shown for the UVES spectrum. The cross shows the adopted NLTE model atmosphere parameters. The $T_{\text {eff }}$ derived from photometry is shown by arrows. 


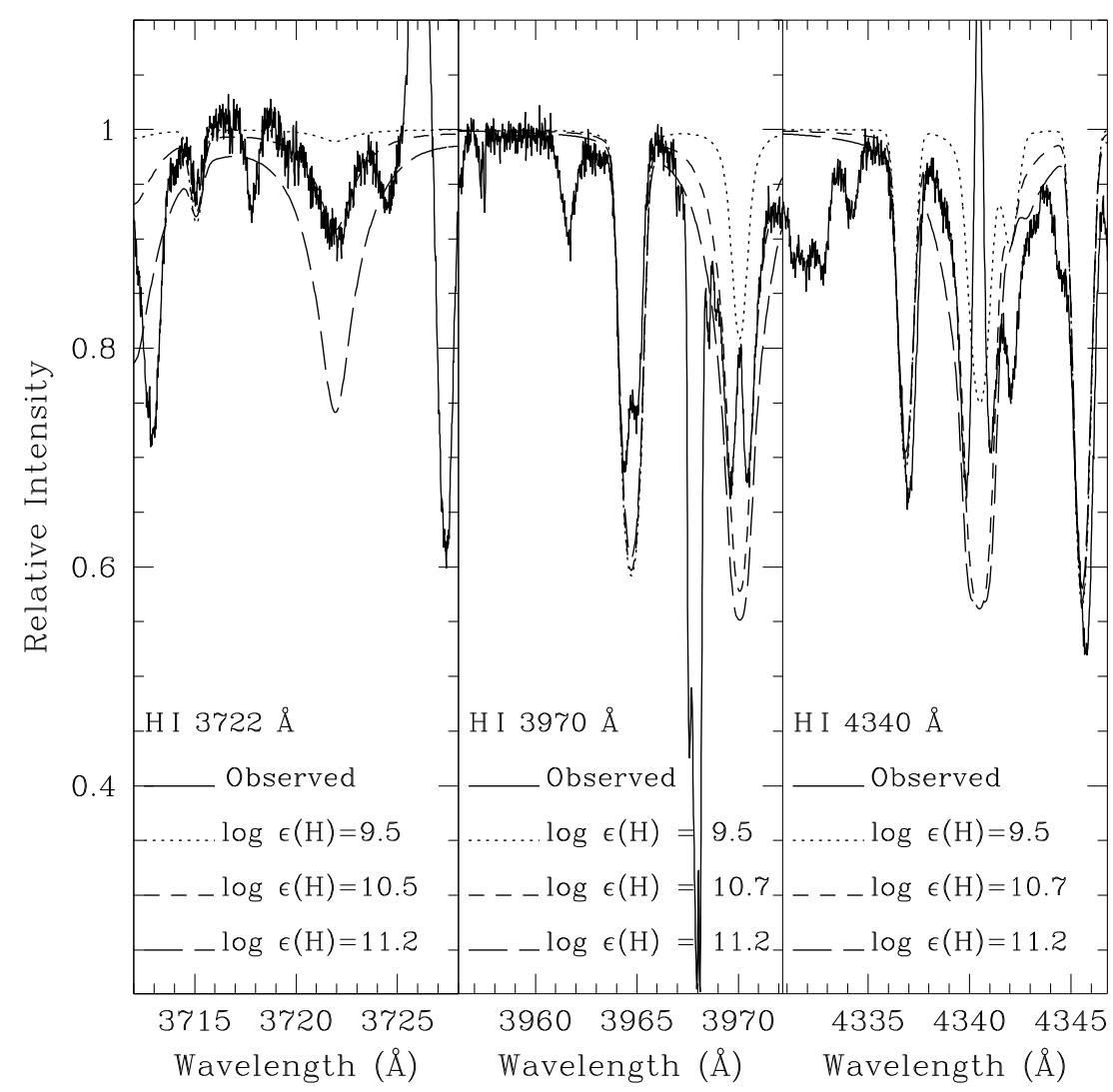

Figure 6. Observed UVES spectrum and theoretical NLTE H I line profiles calculated using the NLTE model $T_{\text {eff }}=25,000 \mathrm{~K}$ and $\log g=2.5$ for three different $\mathrm{H}$ abundances. See the key on the figure.

Table 1

Summary of Atmospheric Parameters

\begin{tabular}{ccccc}
\hline \hline Year & $\begin{array}{c}T_{\text {eff }} \\
(\mathrm{K})\end{array}$ & $\begin{array}{c}\log g \\
(\mathrm{cgs} \text { units })\end{array}$ & $\begin{array}{c}\xi \\
\left(\mathrm{km} \mathrm{s}^{-1}\right)\end{array}$ & $\begin{array}{c}v \sin i \\
\left(\mathrm{~km} \mathrm{~s}^{-1}\right)\end{array}$ \\
\hline 1987 & $19,400 \pm 400$ & $2.10 \pm 0.15$ & $20 \pm 3$ & $20-25$ \\
2002 & $23,000 \pm 300$ & $2.35 \pm 0.15$ & $23 \pm 3$ & $20-27$ \\
2010 & $24,800 \pm 600$ & $2.50 \pm 0.12$ & $24 \pm 3$ & $40 \pm 5$ \\
\hline
\end{tabular}

Similarly to He I, the Hi line profiles are affected by emissions. The H I observed profiles at $3722 \AA$ A $3970 \AA$ and $4340 \AA$ were chosen for estimating the NLTE hydrogen abundance by spectrum synthesis. The line wings of the $3970 \AA$ and $4340 \AA$ profiles are mainly used for this purpose as their cores are severely affected by emissions. The hydrogen model atoms and line-broadening coefficients are from TLUSTY. Observed profiles of the $3722 \AA, 4397 \AA$, and $4340 \AA$ are shown in Figure 6 with predicted non-LTE profiles for a non-LTE atmosphere of $T_{\text {eff }}=25,000 \mathrm{~K}$ and $\log g=2.50$ for three different hydrogen abundances.

The abundance analysis for all elements was conducted for the model atmosphere $\left(T_{\text {eff }}, \log g, \xi\right)=(25,000,2.50$, 24.0). The final photospheric line-by-line non-LTE and/or LTE abundances (including the mean abundance) and the line-to-line scatter are given in Tables 3 and 4 . The lines giving significantly deviant abundances are marked by a question mark and are not included in estimating the mean. The abundance rms errors, due to uncertainty in $T_{\text {eff }}$ and $\log g$, from C II, C III, C IV, N II, N III, $\mathrm{O}$ II, O III, and $\mathrm{Ne}$ II are $0.11,0.14,0.29,0.09,0.12,0.08,0.14$, and 0.13 dex, respectively.

Similarly, we have also conducted the LTE analysis. The LTE TLUSTY models with the LTE line analysis give the final model parameters: $\left(T_{\text {eff }}, \log g, \xi\right)=(24,750,2.65,30.0)$. The LTE abundances for the best LTE TLUSTY model are given in Tables 3 and 4 . The abundance rms errors, due to uncertainty in $T_{\text {eff }}$ and $\log g$, are very similar to those estimated for the appropriate non-LTE model atmosphere.

\subsubsection{Atmospheric Parameters-Summary}

Results of the analysis of the $\mathrm{He}, \mathrm{C}, \mathrm{N}$, and $\mathrm{O}$ lines for the CASPEC, UCLES, and UVES spectra are summarized in Table 1 and shown graphically in Figure 5 where the available loci are plotted together with the $T_{\text {eff }}$ from the $V$-band photometry. The adopted $T_{\text {eff }}$ and $\log g$ for each spectrum is shown by the cross in each panel. For a given spectrum, the cross is a good representation of each available locus and in fair to good agreement with the $T_{\text {eff }}$ from the $V$-band photometry. These spectroscopic analyses show that DY Cen evolved to higher effective temperature and higher surface gravities between 1987 and 2010 following the trend suggested by photometry since early in the twentieth century (Figure 1).

The DY Cen's derived stellar parameters for all three epochs (Figure 7) are plotted on Figure 6 of Saio \& Jeffery (2002) for the merger products. This suggests that DY Cen has evolved at a constant luminosity corresponding to a $0.9 M_{\odot}$ model starting from 0.6-0.5 $M_{\odot}$ CO WDs. DY Cen has become hotter by about $5000 \mathrm{~K}$ in $23 \mathrm{yr}$, i.e., at a rate of about $\log (\mathrm{dTeff} / \mathrm{dt})(K / \mathrm{yr})=$ 2.34. This rate is higher when compared with the rates given by Saio \& Jeffery (2002) in their Figure 7 for the merger products. Rao et al. (2012), based on their radial velocity measurements, noticed that DY Cen is a binary. It is possible that the binary companion, by interaction, is increasing DY Cen's mass loss rate and, hence, enhancing its evolutionary rate as observed by Schönberner (1979). 


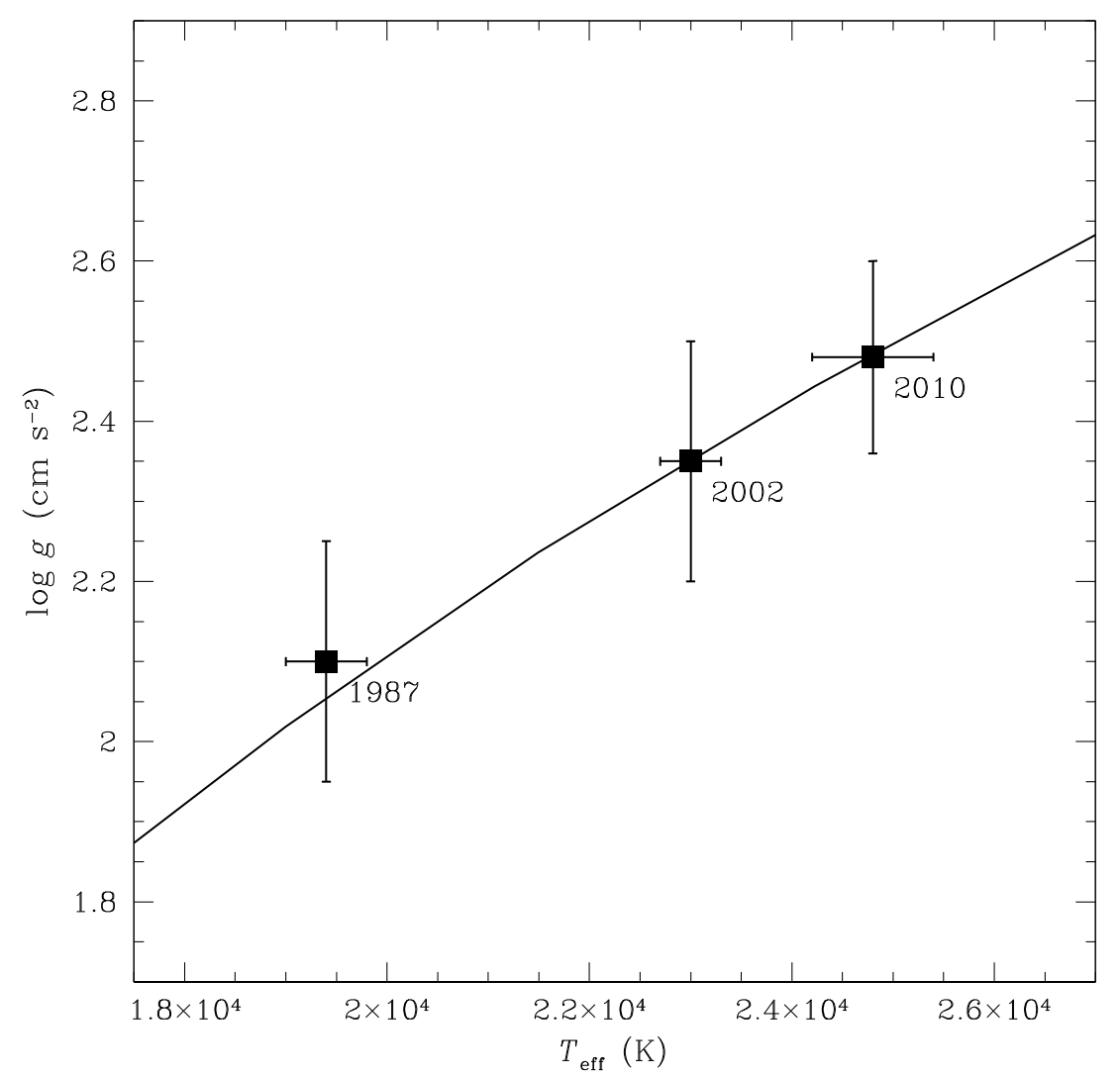

Figure 7. Derived stellar parameters with error bars for all three epochs. Evolutionary track from Figure 6 of Saio \& Jeffery (2002) for the merger product corresponding to a $0.9 M_{\odot}$ model starting from $0.6-0.5 M_{\odot} \mathrm{CO}$ WDs.

\section{CHEMICAL COMPOSITION}

Since the dominant opacity across the optical is provided by neutral $\mathrm{He}$ atoms, the lines of an ion are sensitive to the abundance ratio of that element to helium. Abundances for element $\mathrm{X}-\log \epsilon(\mathrm{X})$-are normalized to $\log \Sigma \mu_{\mathrm{X}} \epsilon(\mathrm{X})=12.15$, where $\mu_{\mathrm{X}}$ is the atomic weight of element $\mathrm{X}$.

With the exception of neon, which is well represented by both the Ne I and Ne II lines, the elements beyond $\mathrm{C}, \mathrm{N}$, and $\mathrm{O}$ provide either no or just a few detectable lines. Thus the accuracy of an elemental abundance depends in large part on secure identifications of stellar lines. In this regard, the higher quality of the UVES and (smoothed) UCLES spectra relative to the CASPEC spectrum has led to several revisions of the line identifications used by JH93 in their abundance analysis.

In the following, selected elements from $\mathrm{H}$ to $\mathrm{Fe}$ are discussed, and recommended abundances are derived. Recommendations are given in Table 5 where we also list the abundances from JH93 (their Table 2). The various RMT cited refer to Moore (1972).

Hydrogen. The $\mathrm{H}$ abundance from select Balmer lines in the UVES spectra is given in Table 3: the mean non-LTE abundance is 10.65 , corresponding to a $\mathrm{H} / \mathrm{He}$ ratio of 0.13 by number. Our analysis of $\mathrm{H} \beta, \mathrm{H} \gamma$, and $\mathrm{H} \delta$ in the CASPEC and UCLES spectra confirm this abundance with $10.6 \pm 0.1$ and $10.7 \pm 0.1$ from CASPEC and UCLES spectra, respectively. These $\mathrm{H}$ abundances are consistent with the LTE value of $10.76 \pm 0.20$ obtained by JH93; the corrections for non-LTE effects are small. With this $\mathrm{H} / \mathrm{He}$ ratio, DY Cen is among the most $\mathrm{H}$-rich of the He-rich H-poor supergiants, including the EHe and RCB stars, as JH93 recognized.
Carbon. Carbon is represented by roughly equal numbers of $\mathrm{C}_{\text {II }}$ and $\mathrm{C}_{\text {III }}$ lines, and in the case of the UVES spectra by two $\mathrm{C}$ IV lines. Examination of the non-LTE abundances from the different ions in Tables 2 and 3 shows consistent results from the ions in the individual spectra. The unweighted means that suggest a quasi-constant abundance across the CASPEC, UCLES, and UVES spectra are 9.54 (CASPEC), 9.41 (UCLES), and 9.74 (UVES) for mean abundances of 9.57 from C II lines and 9.54 from $\mathrm{C}$ III lines. Given the errors of measurement and the line variability, including the appearance of emission components, the apparent variation of the $\mathrm{C}$ abundance is not considered a real effect. We adopt a mean $\mathrm{C}$ abundance of 9.55 or a $\mathrm{C} / \mathrm{He}$ ratio of $1.0 \%$ by number.

Nitrogen. The $\mathrm{N}$ abundance is heavily dependent on the $\mathrm{N}$ II lines, with a single $\mathrm{N}$ III line providing supporting evidence. The two ions give very similar N non-LTE abundances, a comforting result in that the non-LTE corrections are quite different for the two ions. The mean abundances do not change significantly from the CASPEC, UCLES, and UVES spectra: $\log \epsilon(\mathrm{N})=7.78$ is adopted.

Oxygen. For the UVES spectra, the $\mathrm{O}$ abundance is based on roughly equal numbers of $\mathrm{O}$ II and $\mathrm{O}$ III lines with good agreement between the non-LTE abundance from the two ions. The CASPEC and UCLES spectra from the $\mathrm{O}_{\text {II }}$ lines alone give a slightly lower $\mathrm{O}$ abundance than does the value from the UVES spectrum. The mean non-LTE abundance from the three sets of $\mathrm{O}$ II $\operatorname{lines} \log \epsilon(\mathrm{O})=8.87$.

Fluorine. A multiplet-by-multiplet search for F II lines resulted in detections of two lines from RMT 3 (Figure 8): the strongest line, a blend of three components, is present and appears unblended at $3505.6 \AA$; the next strongest line, also a 
Table 2

Measured Equivalent Widths $\left(W_{\lambda}\right)$ and NLTE Photospheric Line Abundances for DY Cen CASPEC and UCLES Spectra

\begin{tabular}{|c|c|c|c|c|c|}
\hline \multirow[t]{2}{*}{ Line } & \multirow{2}{*}{$\begin{array}{c}\chi \\
(\mathrm{eV})\end{array}$} & \multirow[b]{2}{*}{$\log g f$} & \multirow{2}{*}{$\begin{array}{c}W_{\lambda}: \text { CASPEC, AAT/UCLES } \\
(\mathrm{m} \AA)\end{array}$} & \multicolumn{2}{|c|}{$\log \epsilon(\mathrm{X})$} \\
\hline & & & & CASPEC $^{\mathrm{a}}$ & AAT/UCLES ${ }^{\mathrm{b}}$ \\
\hline Н г $\lambda 4101.734$ & 10.199 & -0.753 & Synth & 10.75 & 10.52 \\
\hline Н г $\lambda 4340.462$ & 10.199 & -0.447 & Synth & 10.72 & 10.63 \\
\hline Н г $\lambda 4861.323$ & 10.199 & -0.020 & Synth & 10.58 & 10.70 \\
\hline Mean... & $\ldots$ & $\cdots$ & $\cdots$ & $10.70 \pm 0.10$ & $10.60 \pm 0.10$ \\
\hline C II $\lambda 4017.272$ & 22.899 & -1.031 & $85, \cdots$ & 9.65 & $\ldots$ \\
\hline C II $\lambda 4021.166$ & 22.899 & -1.333 & 35,40 & 9.46 & 9.64 \\
\hline C II $\lambda 4321.657$ & 23.116 & -0.901 & $\cdots, 56$ & $\cdots$ & 9.45 \\
\hline $\mathrm{C}_{\text {II }} \lambda 4323.107$ & 23.114 & -1.105 & 49,32 & 9.49 & 9.38 \\
\hline $\mathrm{C}_{\text {II }} \lambda 4325.832$ & 23.119 & -0.373 & & & \\
\hline C II $\lambda 4326.164$ & 23.116 & -0.407 & 234,184 & 9.48 & 9.37 \\
\hline $\mathrm{C}_{\text {II }} \lambda 4372.375$ & 24.656 & $+0.057^{\mathrm{c}}$ & & & \\
\hline C II $\lambda 4372.501$ & 24.658 & $+0.272^{\mathrm{c}}$ & $244, \cdots$ & 9.56 & $\ldots$ \\
\hline $\mathrm{C}_{\text {II }} \lambda 4374.281$ & 24.654 & $+0.660^{\mathrm{d}}$ & 302,225 & 9.69 & 9.32 \\
\hline $\mathrm{C}_{\text {II }} \lambda 4376.582$ & 24.656 & $+0.380^{\mathrm{d}}$ & $184, \cdots$ & 9.38 & $\cdots$ \\
\hline Mean... & $\cdots$ & $\cdots$ & $\cdots$ & $9.53 \pm 0.11$ & $9.43 \pm 0.13$ \\
\hline $\mathrm{C}_{\text {III }} \lambda 4162.877$ & 40.064 & +0.218 & $\cdots, 85$ & $\ldots$ & 9.52 \\
\hline C III $\lambda 4186.900$ & 40.010 & +0.918 & 52,150 & 9.63 & 9.43 \\
\hline C III $\lambda 4647.418$ & 29.535 & +0.070 & 261,483 & 9.40 & 9.19 \\
\hline C III $\lambda 4651.473$ & 29.535 & -0.629 & $153, \cdots$ & 9.62 & $\ldots$ \\
\hline $\mathrm{C}_{\text {III }} \lambda 4665.860$ & 38.226 & +0.044 & $\cdots, 90$ & $\ldots$ & 9.43 \\
\hline Mean... & $\cdots$ & $\cdots$ & $\cdots$ & $9.55 \pm 0.13$ & $9.39 \pm 0.14$ \\
\hline N II $\lambda 4227.736$ & 21.599 & -0.061 & 74,61 & 7.68 & 7.67 \\
\hline N II $\lambda 4447.030$ & 20.409 & +0.221 & 216,150 & 7.86 & 7.62 \\
\hline N II $\lambda 4601.478$ & 18.466 & -0.452 & 192,241 & 7.82 & 8.18 \\
\hline N II $\lambda 4607.153$ & 18.462 & -0.522 & 174,189 & 7.80 & 8.03 \\
\hline N II $\lambda 4613.868$ & 18.466 & -0.692 & $\cdots, 163$ & $\cdots$ & 8.08 \\
\hline N II $\lambda 4630.539$ & 18.483 & +0.080 & 288,300 & 7.74 & 7.92 \\
\hline N II $\lambda 4643.086$ & 18.483 & -0.371 & 113,198 & $7.34 ?$ & 7.92 \\
\hline N II $\lambda 4788.138$ & 20.654 & -0.366 & 46,48 & 7.47 & 7.62 \\
\hline Mean... & $\cdots$ & $\cdots$ & $\cdots$ & $7.67 \pm 0.20$ & $7.88 \pm 0.21$ \\
\hline N III $\lambda 4200.070$ & 36.856 & +0.250 & 12,30 & $8.24 ?$ & 7.85 \\
\hline O II $\lambda 4104.724$ & 25.837 & -0.302 & & & \\
\hline O II $\lambda 4104.990$ & 25.837 & -0.015 & 180,242 & 8.84 & 9.33 \\
\hline O II $\lambda 4153.298$ & 25.837 & +0.053 & $176, \ldots$ & 8.95 & $\ldots$ \\
\hline O II $\lambda 4317.139$ & 22.966 & -0.386 & 262,344 & 8.79 & 8.85 \\
\hline O II $\lambda 4319.630$ & 22.979 & -0.380 & 241,357 & 8.71 & 8.88 \\
\hline O II $\lambda 4336.859$ & 22.979 & -0.763 & 207,240 & 8.99 & 8.93 \\
\hline O II $\lambda 4345.560$ & 22.979 & -0.346 & 323,376 & 8.97 & 8.92 \\
\hline O II $\lambda 4349.426$ & 22.999 & +0.060 & 384,550 & 8.74 & 8.94 \\
\hline O II $\lambda 4366.895$ & 22.999 & -0.348 & 291, 396 & 8.85 & 8.96 \\
\hline O II $\lambda 4414.899$ & 23.442 & +0.172 & 330,514 & 8.47 & 8.62 \\
\hline O II $\lambda 4416.975$ & 23.419 & -0.077 & 281,418 & 8.56 & 8.63 \\
\hline O II $\lambda 4452.378$ & 23.442 & -0.788 & 152,209 & 8.76 & 8.72 \\
\hline O II $\lambda 4661.632$ & 22.979 & -0.278 & 225,373 & 8.56 & 8.79 \\
\hline O II $\lambda 4673.733$ & 22.979 & -1.090 & 153,245 & 9.04 & 9.19 \\
\hline O II $\lambda 4676.235$ & 22.999 & -0.394 & 244,358 & 8.75 & 8.86 \\
\hline O II $\lambda 4696.353$ & 22.999 & -1.380 & 37,95 & 8.41 & 8.80 \\
\hline O II $\lambda 4705.346$ & 26.249 & +0.477 & $179, \ldots$ & 8.16 & $\ldots$ \\
\hline O II $\lambda 4906.830$ & 26.305 & -0.161 & 112,235 & 8.47 & 8.53 \\
\hline O II $\lambda 4924.529$ & 26.305 & +0.074 & 175,300 & 8.53 & 8.45 \\
\hline O II $\lambda 4941.072$ & 26.554 & -0.053 & $88, \cdots$ & 9.07 & $\ldots$ \\
\hline O II $\lambda 4943.005$ & 26.561 & +0.239 & $152, \cdots$ & 9.25 & $\cdots$ \\
\hline Mean... & $\cdots$ & $\ldots$ & $\ldots$ & $8.74 \pm 0.27$ & $8.84 \pm 0.22$ \\
\hline
\end{tabular}

Notes.

${ }^{\mathrm{a}}\left(T_{\mathrm{eff}}, \log g, \xi\right)=(19,400,2.10,20.0)$.

b $\left(T_{\text {eff }}, \log g, \xi\right)=(23,000,2.35,23.0)$.

c Yan et al. (1987).

${ }^{d}$ Wiese et al. (1966). 
Table 3

Measured Equivalent Widths $\left(W_{\lambda}\right)$ and NLTE/LTE Photospheric Line Abundances for the DY Cen UVES Spectrum

\begin{tabular}{|c|c|c|c|c|c|}
\hline \multirow[t]{2}{*}{ Line } & \multirow{2}{*}{$\begin{array}{c}\chi \\
(e V)\end{array}$} & \multirow[b]{2}{*}{$\log g f$} & \multirow{2}{*}{$\begin{array}{c}W_{\lambda} \\
(\mathrm{m} \AA)\end{array}$} & \multicolumn{2}{|c|}{$\log \epsilon(\mathrm{X})$} \\
\hline & & & & NLTE $^{\mathrm{a}}$ & $\mathrm{LTE}^{\mathrm{b}}$ \\
\hline Н г $\lambda 3721.939$ & 10.199 & -1.975 & Synth & 10.52 & \\
\hline Н І $\lambda 3970.072$ & 10.199 & -0.993 & Synth & 10.72 & \\
\hline Н І $\lambda 4340.462$ & 10.199 & -0.447 & Synth & 10.72 & \\
\hline Mean... & $\cdots$ & $\cdots$ & $\cdots$ & $10.65 \pm 0.12$ & $10.76 \pm 0.04$ \\
\hline $\mathrm{C}_{\text {II }} \lambda 3581.757$ & 22.529 & -1.643 & 36 & 10.10 & 10.15 \\
\hline $\mathrm{C}_{\text {II }} \lambda 3952.057$ & 24.278 & $-0.210^{\mathrm{c}}$ & 152 & 9.82 & 9.85 \\
\hline $\mathrm{C}_{\text {II }} \lambda 3977.250$ & 24.372 & $-0.500^{\mathrm{c}}$ & 66 & 9.58 & 9.62 \\
\hline C II $\lambda 3980.317$ & 24.373 & $-0.210^{\mathrm{c}}$ & 105 & 9.57 & 9.60 \\
\hline $\mathrm{C}_{\text {II }} \lambda 4017.272$ & 22.899 & $-1.031^{\mathrm{c}}$ & 74 & 9.83 & 9.90 \\
\hline C II $\lambda 4021.166$ & 22.899 & $-1.333^{c}$ & 39 & 9.79 & 9.86 \\
\hline $\mathrm{C}_{\text {II }} \lambda 4313.106$ & 23.116 & -0.373 & 202 & 9.96 & 10.01 \\
\hline C II $\lambda 4321.657$ & 23.116 & -0.901 & 55 & 9.59 & 9.65 \\
\hline $\mathrm{C}_{\text {II }} \lambda 4323.107$ & 23.114 & -1.105 & 39 & 9.62 & 9.68 \\
\hline С II $\lambda 4374.281$ & 24.654 & +0.660 & 353 & 10.07 & 10.01 \\
\hline Mean... & $\ldots$ & $\ldots$ & $\cdots$ & $9.79 \pm 0.20$ & $9.83 \pm 0.19$ \\
\hline C III $\lambda 3885.938$ & 39.852 & +0.205 & & & \\
\hline $\mathrm{C}_{\text {III }} \lambda 3886.145$ & 39.852 & -0.698 & 152 & 9.62 & 9.77 \\
\hline $\mathrm{C}_{\text {III }} \lambda 4056.061$ & 40.197 & +0.265 & 160 & 9.66 & 9.72 \\
\hline $\mathrm{C}_{\text {III }} \lambda 4162.877$ & 40.064 & +0.218 & 173 & 9.81 & 9.92 \\
\hline $\mathrm{C}_{\text {III }} \lambda 4186.900$ & 40.010 & +0.918 & 246 & 9.50 & 9.68 \\
\hline $\mathrm{C}_{\text {III }} \lambda 4382.897$ & 39.852 & -0.778 & & & \\
\hline $\mathrm{C}_{\text {III }} \lambda 4383.533$ & 39.852 & -1.255 & 66 & 9.67 & 9.65 \\
\hline $\mathrm{C}_{\text {III }} \lambda 5826.420$ & 40.197 & +0.416 & 160 & 9.70 & 9.93 \\
\hline C III $\lambda 6350.770$ & 40.197 & -0.882 & 17 & 9.83 & 9.80 \\
\hline Mean... & $\cdots$ & $\cdots$ & $\cdots$ & $9.68 \pm 0.11$ & $9.78 \pm 0.11$ \\
\hline $\mathrm{C}_{\mathrm{IV}} \lambda 5801.310$ & 37.548 & -0.194 & 42: & 9.72 & 9.80 \\
\hline $\mathrm{C}_{\text {IV }} \lambda 5811.970$ & 37.548 & -0.495 & $24:$ & 9.86 & 9.60 \\
\hline Mean... & $\ldots$ & $\ldots$ & $\cdots$ & $9.79 \pm 0.10$ & $9.70 \pm 0.14$ \\
\hline N II $\lambda 3437.145$ & 18.497 & -0.436 & 112 & 7.97 & 8.13 \\
\hline N II $\lambda 3955.851$ & 18.466 & -0.813 & 78 & 8.01 & 8.22 \\
\hline N II $\lambda 3994.997$ & 18.497 & +0.208 & 349 & $8.32 ?$ & 8.42 \\
\hline $\mathrm{N}_{\text {II }} \lambda 4035.081$ & 23.124 & $+0.623^{\mathrm{c}}$ & 85 & 7.75 & 7.87 \\
\hline $\mathrm{N}_{\text {II }} \lambda 4041.310$ & 23.142 & $+0.853^{\mathrm{c}}$ & 135 & 7.82 & 7.93 \\
\hline N II $\lambda 4043.532$ & 23.132 & $+0.743^{\mathrm{c}}$ & 78 & 7.58 & 7.70 \\
\hline $\mathrm{N}_{\text {II }} \lambda 4171.595$ & 23.196 & $+0.280^{\mathrm{c}}$ & 35 & 7.64 & 7.77 \\
\hline $\mathrm{N}_{\text {II }} \lambda 4176.159$ & 23.196 & $+0.600^{c}$ & 65 & 7.64 & 7.76 \\
\hline N II $\lambda 4227.736$ & 21.600 & -0.061 & 50 & 7.78 & 7.93 \\
\hline N II $\lambda 4241.755$ & 23.242 & $+0.210^{\mathrm{c}}$ & & & \\
\hline N II $\lambda 4241.786$ & 23.246 & $+0.713^{\mathrm{c}}$ & 103 & 7.69 & 7.92 \\
\hline $\mathrm{N}_{\text {II }} \lambda 4432.736$ & 23.415 & $+0.580^{\mathrm{c}}$ & 80 & 7.83 & 7.95 \\
\hline Mean... & $\cdots$ & $\cdots$ & $\cdots$ & $7.77 \pm 0.14$ & $7.92 \pm 0.16$ \\
\hline N III $\lambda 4200.070$ & 36.856 & +0.250 & 42 & 7.78 & 8.42 \\
\hline O II $\lambda 3305.003$ & 25.849 & -0.723 & 85 & $9.55 ?$ & 9.16 \\
\hline O II $\lambda 3306.451$ & 25.837 & -0.740 & 80 & $9.53 ?$ & 9.14 \\
\hline O II $\lambda 3377.146$ & 25.286 & -0.342 & 168 & 8.89 & 9.15 \\
\hline O II $\lambda 3390.209$ & 25.286 & -0.044 & 212 & 8.92 & 9.07 \\
\hline O II $\lambda 3407.223$ & 28.510 & -1.121 & & & \\
\hline O II $\lambda 3407.276$ & 28.512 & +0.025 & 130 & 9.37 & 9.30 \\
\hline O II $\lambda 3409.706$ & 28.512 & -0.167 & & & \\
\hline O II $\lambda 3409.760$ & 28.512 & -1.121 & 65 & 9.03 & 9.02 \\
\hline O II $\lambda 3739.761$ & 26.305 & -0.427 & 175 & 8.85 & 9.19 \\
\hline O II $\lambda 3762.465$ & 26.305 & -0.580 & 154 & 8.92 & 9.23 \\
\hline O II $\lambda 3882.194$ & 25.664 & -0.035 & 290 & $9.48 ?$ & 9.15 \\
\hline O II $\lambda 3893.518$ & 25.638 & -1.589 & 32 & 9.19 & \\
\hline O II $\lambda 3907.455$ & 25.649 & -0.925 & 105 & 9.39 & 9.11 \\
\hline O II $\lambda 3911.958$ & 25.661 & +0.000 & & & \\
\hline O II $\lambda 3912.117$ & 25.661 & -0.888 & 342 & $9.52 ?$ & 9.16 \\
\hline O II $\lambda 3945.038$ & 23.419 & -0.727 & 330 & 8.91 & $9.47 ?$ \\
\hline
\end{tabular}


Table 3

(Continued)

\begin{tabular}{|c|c|c|c|c|c|}
\hline \multirow[t]{2}{*}{ Line } & \multirow{2}{*}{$\begin{array}{c}\chi \\
(\mathrm{eV})\end{array}$} & \multirow[b]{2}{*}{$\log g f$} & \multirow{2}{*}{$\begin{array}{c}W_{\lambda} \\
(\mathrm{m} \AA) \\
\end{array}$} & \multicolumn{2}{|c|}{$\log \epsilon(\mathrm{X})$} \\
\hline & & & & NLTE $^{a}$ & $\mathrm{LTE}^{\mathrm{b}}$ \\
\hline O II $\lambda 3954.362$ & 23.419 & -0.396 & 423 & 8.80 & $9.51 ?$ \\
\hline O II $\lambda 3973.256$ & 23.441 & -0.015 & 585 & 8.83 & \\
\hline O II $\lambda 3982.714$ & 23.441 & -0.703 & 340 & 8.90 & $9.50 ?$ \\
\hline O II $\lambda 4185.440$ & 28.357 & +0.604 & 240 & 8.96 & 8.84 \\
\hline O II $\lambda 4189.581$ & 28.360 & -0.828 & & & \\
\hline O II $\lambda 4189.794$ & 28.360 & +0.716 & 253 & 8.89 & 8.77 \\
\hline O II $\lambda 4192.512$ & 28.509 & -0.470 & 48 & 8.83 & 8.89 \\
\hline O II $\lambda 4196.273$ & 28.512 & -1.425 & & & \\
\hline O II $\lambda 4196.697$ & 28.512 & -0.726 & 63 & 9.13 & 9.20 \\
\hline O II $\lambda 4327.460$ & 28.509 & +0.057 & & & \\
\hline O II $\lambda 4327.849$ & 28.509 & -1.090 & 140 & 8.93 & 8.93 \\
\hline O II $\lambda 4336.859$ & 22.979 & -0.763 & 345 & 9.16 & $9.58 ?$ \\
\hline O II $\lambda 4345.560$ & 22.979 & -0.346 & 465 & 9.20 & \\
\hline O II $\lambda 4349.426$ & 22.998 & +0.060 & 687 & 9.33 & \\
\hline O II $\lambda 4366.895$ & 22.999 & -0.348 & 570 & $9.48 ?$ & \\
\hline O II $\lambda 4395.935$ & 26.248 & -0.167 & 190 & 8.93 & 8.99 \\
\hline O II $\lambda 4405.978$ & 26.248 & -1.300 & 29 & 8.98 & 8.99 \\
\hline O II $\lambda 4414.899$ & 23.441 & +0.172 & 665 & 9.10 & \\
\hline O II $\lambda 4416.975$ & 23.419 & -0.077 & 530 & 9.03 & \\
\hline O II $\lambda 4452.378$ & 23.442 & -0.788 & 265 & 9.04 & 9.30 \\
\hline O II $\lambda 4443.010$ & 28.358 & -0.047 & 156 & 9.17 & 9.13 \\
\hline O II $\lambda 4448.191$ & 28.361 & +0.083 & 188 & 9.22 & 9.17 \\
\hline O II $\lambda 6641.031$ & 23.419 & -0.884 & 394 & $9.55 ?$ & \\
\hline O II $\lambda 6721.388$ & 23.441 & -0.610 & 582 & $9.64 ?$ & \\
\hline Mean... & $\cdots$ & $\cdots$ & $\cdots$ & $9.03 \pm 0.17$ & $9.09 \pm 0.14$ \\
\hline O III $\lambda 3312.329$ & 33.151 & -0.644 & 36 & 8.93 & 9.26 \\
\hline O III $\lambda 3340.765$ & 33.182 & -0.482 & 56 & 9.04 & 9.47 \\
\hline O III $\lambda 3430.568$ & 37.250 & -0.902 & 18 & 9.15 & 9.32 \\
\hline O III $\lambda 3444.052$ & 37.250 & -0.427 & 34 & 9.02 & 9.29 \\
\hline O III $\lambda 3707.272$ & 37.234 & -0.121 & 31 & 8.70 & 8.83 \\
\hline O III $\lambda 3715.086$ & 37.249 & +0.149 & 56 & 8.82 & 9.04 \\
\hline O III $\lambda 3754.696$ & 33.150 & -0.099 & 126 & 8.83 & 9.26 \\
\hline O III $\lambda 3757.232$ & 33.135 & -0.452 & 86 & 8.88 & 9.16 \\
\hline O III $\lambda 3759.875$ & 33.182 & +0.162 & 157 & 8.73 & 9.22 \\
\hline O III $\lambda 3774.026$ & 33.150 & -0.601 & 60 & 8.81 & 9.08 \\
\hline O III $\lambda 3810.985$ & 33.182 & -1.810 & 8 & 8.79 & 8.88 \\
\hline O III $\lambda 3961.573$ & 38.011 & +0.314 & 60 & 9.05 & 9.04 \\
\hline Mean... & $\cdots$ & $\cdots$ & $\cdots$ & $8.90 \pm 0.14$ & $9.15 \pm 0.19$ \\
\hline
\end{tabular}

Notes.

${ }^{\mathrm{a}}\left(T_{\mathrm{eff}}, \log g, \xi\right)=(25,000,2.50,24.0)$.

${ }^{\mathrm{b}}\left(T_{\mathrm{eff}}, \log g, \xi\right)=(24,750,2.65,30.0)$.

${ }^{\mathrm{c}}$ Kurucz $g f$-value.

blend of three components, at $3503.1 \AA$ is present; the multiplet's weakest line at $3501.4 \AA$ falls in the wing of a He I line. Lines from other multiplets are masked by stronger lines from other contributing ions. Table 4 shows that the two F II blends give similar LTE abundances with $g f$-values from the NIST Web site. The abundance $\log \epsilon(\mathrm{F})=7.0$ is adopted. (F I lines are absent as expected-see Pandey's (2006) collection of spectra of extreme helium stars.)

Neon. Neon is well represented by both $\mathrm{Ne}$ I and Ne II lines. Model atoms in SYNSPEC allow us to compute non-LTE (and LTE) Ne abundances for both the neutral and ionized lines.

Neutral $\mathrm{Ne}$ is represented in DY Cen by several multiplets in the red. Five clean lines are given in Table 4 with $g f$-values from the NIST Web site. The equivalent widths of these lines are substantially smaller than those reported by $\mathrm{JH} 93$, and the stellar profiles appear to be composed of a blend of two equal components or contaminated by weak central emission. The mean $\mathrm{Ne}$ abundance from $\mathrm{Ne}$ I lines is 8.7 and 9.5 from nonLTE and LTE analyses, respectively.

Ionized Ne provides many lines, and a large selection of the cleanest lines is provided in Table 4 . The $g f$-values are taken from the NIST website if available or else from Kurucz's website. For the UVES spectrum, the mean neon abundances from the Ne II lines are $8.4 \pm 0.2$ (LTE) and $8.0 \pm 0.1$ (nonLTE). These mean values are substantially lower than those from the Ne I lines: the difference is 0.7 dex for the non-LTE analyses. The $\mathrm{Ne}$ abundance from the $\mathrm{Ne}$ I lines is confirmed by reanalysis of the Ne I lines measured by Rao et al. (1993) and listed by JH93 off a spectrum from 1989.

Magnesium. In the bandpass of the UVES spectrum, the sole signature of magnesium is the $\mathrm{Mg}$ II $4481 \AA$ feature. Atomic data for the feature composed of three unresolved lines are from 


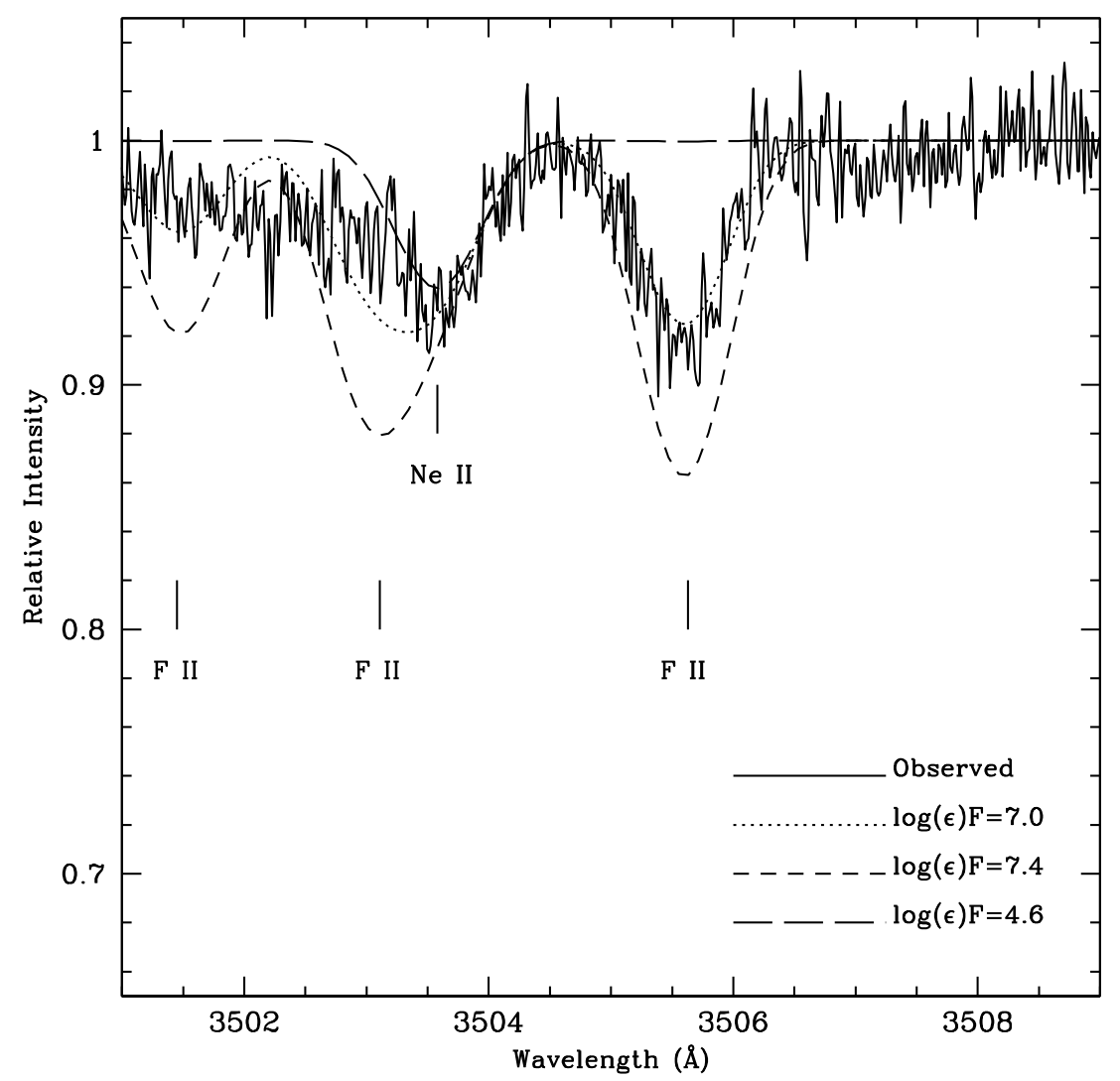

Figure 8. Observed UVES spectrum and theoretical F II line profiles calculated using the LTE model $T_{\text {eff }}=24,750 \mathrm{~K}$ and $\log g=2.65$ for three different $\mathrm{F}$ abundances. See the key on the figure.

Kelleher \& Podobedova's (2008c) critical evaluation which also appears on the NIST Web site. The Mg abundances from the UVES spectrum are $\log \epsilon(\mathrm{Mg})=6.8$ and 7.1 for non-LTE and LTE analyses, respectively.

The $4481 \AA$ feature is also present on the CASPEC and UCLES spectra where its equivalent width is $256 \mathrm{~m} \AA$ and $176 \mathrm{~m} \AA$, respectively, to be compared with $154 \mathrm{~m} \AA$ from the UVES spectrum. The feature when analyzed with the appropriate model atmosphere (Table 1 ) gives very similar nonLTE Mg abundances: namely, 6.70, 6.67, and 6.76 from the CASPEC, UCLE,S and UVES spectra, respectively.

Aluminum. The aluminum abundance must be determined from the few available Al III lines with $\log g f$-values taken from Kelleher \& Podobedova (2008a)'s compilation, except that values for RMT 8 at $4480 \AA$ not included by them are taken from Kurucz. Al II and A IV lines are not expected to be detectable in the available spectra, an expectation confirmed by examination of the spectra.

For the UVES spectra, the Al abundance comes from the four features in Table 4. The Al LTE abundances from three detected lines are 6.07 from $3601.6 \AA, 7.70$ from $5722.7 \AA$ and 6.22 from the blend at $4480 \AA$. The large discrepancy between the $3601 \AA$ and $5722 \AA$ lines comes from two well-resolved lines and no known blend for either line. This discrepancy suggests serious non-LTE effects are present. A feature at $4149.9 \AA$ is absent and sets an abundance upper limit of 5.1, a limit more than 1 dex below the abundance provided by the 3601.6 and $4480 \AA$ lines. The $4149.9 \AA$ feature is also not detectable on the CASPEC and UCLES spectra. Clearly, this limited set of Al III lines gives apparently inconsistent $\mathrm{Al}$ abundances. Our adopted abundance is 6.1 based on the consistent 3601.6 and $4480 \AA$ features.
JH93 chose three Al III features from two multiplets with a third multiplet rejected because of a blend with an $\mathrm{O}$ II line. In addition to the $4480 \AA$ feature, they adopted RMT 3 with a line at $4512.6 \AA$ and a blend near $4529 \AA$. Remeasurement and reanalysis of these three features from CASPEC and UCLES spectra confirms the 1993 result that they give consistent $\mathrm{Al}$ abundances and suggests that our adopted abundance is a reasonable choice.

Silicon. At the atmospheric conditions prevailing from 1987 to 2010, one expects to see lines from the ions $\mathrm{Si}^{+}, \mathrm{Si}^{2+}$ and $\mathrm{Si}^{3+}$ depending on wavelength coverage of available spectra. Atomic data, including $g f$-values, are taken from the critical evaluation by Kelleher \& Podobedova (2008b).

Si II. The only Si II lines on the CASPEC spectrum are from RMT 3 at $4130.89 \AA$ and $4128.05 \AA$ with $\log g f$-values of 0.57 and 0.36 , respectively. JH93 reported these as absorption lines with equivalent widths of $273 \mathrm{~m} \AA$ and $268 \mathrm{~m} \AA$, respectively. Inspection of these lines on the UVES spectra shows that both are blended to the red with a stronger $\mathrm{O}$ II line; the $\mathrm{Si}$ II equivalent widths attributed to the CASPEC spectrum probably refer to the blend of Si II and O II lines. Synthesis of the blends in the UVES spectrum gives the non-LTE Si abundance of 8.4 from both lines.

An advantage of the UVES spectra over the CASPEC and UCLES spectra is that greater wavelength coverage of the red spectrum includes Si II lines from RMT 2 (6347 $\AA$ and $6371 \AA$ ) and RMT 4 (5978 $\AA$ and $5957 \AA$ ). These lines are in emission and not in absorption; the upper term of RMT 2 is the lower term for RMT 4. Application of LTE or non-LTE to absorption lines at $4128 \AA$ and $4130 \AA$ is of suspect validity given that these red multiplets appear in emission. 
Table 4

Measured Equivalent Widths $\left(W_{\lambda}\right)$ and NLTE/LTE Photospheric Line Abundances of F, Ne, and Heavier Elements for the DY Cen UVES Spectrum

\begin{tabular}{|c|c|c|c|c|c|}
\hline \multirow[t]{2}{*}{ Line } & \multirow{2}{*}{$\begin{array}{c}\chi \\
(\mathrm{eV})\end{array}$} & \multirow[b]{2}{*}{$\log g f$} & \multirow{2}{*}{$\begin{array}{c}W_{\lambda} \\
(\mathrm{m} \AA)\end{array}$} & \multicolumn{2}{|c|}{$\log \epsilon(\mathrm{X})$} \\
\hline & & & & NLTE $^{\mathrm{a}}$ & $\mathrm{LTE}^{\mathrm{b}}$ \\
\hline$F_{\text {II }} \lambda 3502.840$ & 25.102 & -0.400 & & & \\
\hline$F_{\text {II }} \lambda 3502.964$ & 25.102 & +0.187 & & & \\
\hline$F_{\text {II }} \lambda 3503.109$ & 25.102 & +0.391 & 42 & & 6.90 \\
\hline$F_{\text {II }} \lambda 3505.368$ & 25.104 & -0.757 & & & \\
\hline F II $\lambda 3505.513$ & 25.104 & +0.090 & & & \\
\hline$F_{\text {II }} \lambda 3505.628$ & 25.104 & +0.676 & 70 & & 7.05 \\
\hline Mean... & $\ldots$ & $\cdots$ & $\ldots$ & & $6.98 \pm 0.11$ \\
\hline $\mathrm{Ne}_{\mathrm{I}} \lambda 6143.063$ & 16.619 & -0.100 & 141 & 8.76 & 9.41 \\
\hline $\mathrm{Ne}_{\mathrm{I}} \lambda 6163.594$ & 16.715 & -0.620 & 70 & 8.70 & 9.54 \\
\hline $\mathrm{Ne}_{\mathrm{I}} \lambda 6266.495$ & 16.715 & -0.370 & 84 & 8.53 & 9.39 \\
\hline $\mathrm{Ne}_{\mathrm{I}} \lambda 6334.428$ & 16.619 & -0.320 & 115 & 8.87 & 9.50 \\
\hline $\mathrm{Ne}$ I $\lambda 6402.246$ & 16.619 & +0.330 & 245 & 8.63 & 9.44 \\
\hline Mean... & $\ldots$ & $\ldots$ & $\ldots$ & $8.70 \pm 0.13$ & $9.46 \pm 0.06$ \\
\hline Ne II $\lambda 3309.739$ & 27.783 & -0.990 & 30 & 8.09 & 8.36 \\
\hline Ne II $\lambda 3323.734$ & 27.783 & +0.030 & 132 & 7.87 & 8.47 \\
\hline Ne II $\lambda 3327.152$ & 27.233 & -0.220 & 130 & 7.92 & 8.58 \\
\hline $\mathrm{Ne}$ II $\lambda 3334.836$ & 27.169 & +0.380 & 235 & 7.72 & 8.61 \\
\hline $\mathrm{Ne}$ II $\lambda 3344.396$ & 27.270 & -0.300 & 140 & 8.05 & 8.74 \\
\hline $\mathrm{Ne}$ II $\lambda 3345.454$ & 30.549 & -0.030 & 73: & 8.08 & 8.69 \\
\hline $\mathrm{Ne}$ II $\lambda 3357.820$ & 30.927 & -0.290 & 37 & 8.15 & 8.59 \\
\hline $\mathrm{Ne}$ II $\lambda 3378.217$ & 27.659 & -0.240 & 72 & 7.79 & 8.29 \\
\hline Ne II $\lambda 3388.419$ & 31.185 & +0.360 & 54 & 7.93 & 8.23 \\
\hline Ne II $\lambda 3417.689$ & 31.121 & +0.350 & 66 & 8.22 & 8.37 \\
\hline Ne II $\lambda 3453.068$ & 31.185 & -0.480 & 30 & 8.11 & 8.44 \\
\hline Ne II $\lambda 3481.933$ & 27.783 & -0.290 & 102 & 7.97 & 8.28 \\
\hline $\mathrm{Ne}$ II $\lambda 3542.845$ & 31.362 & +0.130 & 82 & 7.93 & 8.48 \\
\hline Ne II $\lambda 3557.803$ & 27.859 & -1.140 & 25 & 8.07 & 8.34 \\
\hline $\mathrm{Ne}$ II $\lambda 3565.826$ & 31.362 & -0.330 & 32 & 8.01 & 8.38 \\
\hline Ne II $\lambda 3568.500$ & 30.549 & +0.330 & 136 & 7.88 & 8.51 \\
\hline $\mathrm{Ne}$ II $\lambda 3571.230$ & 31.362 & -0.320 & 45 & 8.17 & 8.55 \\
\hline $\mathrm{Ne}$ II $\lambda 3574.611$ & 30.549 & +0.170 & 126 & 7.99 & 8.60 \\
\hline $\mathrm{Ne}$ II $\lambda 3628.036$ & 31.512 & -0.320 & 20 & 7.95 & 8.18 \\
\hline $\mathrm{Ne}$ II $\lambda 3643.928$ & 27.783 & -0.590 & 56 & 7.82 & 8.21 \\
\hline Ne II $\lambda 3664.073$ & 27.169 & -0.250 & 172 & 7.92 & 8.58 \\
\hline $\mathrm{Ne}$ II $\lambda 3694.212$ & 27.169 & +0.090 & 233 & 7.75 & 8.51 \\
\hline Ne II $\lambda 3709.621$ & 27.233 & -0.340 & 170 & 7.93 & 8.61 \\
\hline Ne II $\lambda 3766.258$ & 27.233 & -0.430 & 156 & 7.92 & 8.57 \\
\hline Ne II $\lambda 3777.134$ & 27.270 & -0.440 & 190 & 8.06 & 8.76 \\
\hline Ne II $\lambda 4150.690$ & 34.644 & $-0.030^{\mathrm{c}}$ & 22 & 8.16 & 8.46 \\
\hline $\mathrm{Ne}$ II $\lambda 4217.169$ & 34.609 & $+0.090^{\mathrm{c}}$ & 15 & 7.86 & 8.16 \\
\hline Ne II $\lambda 4219.745$ & 34.609 & $+0.750^{\mathrm{c}}$ & 96 & 8.17 & 8.57 \\
\hline Ne II $\lambda 4231.532$ & 34.619 & $-0.080^{\mathrm{c}}$ & & & \\
\hline Ne II $\lambda 4231.636$ & 34.619 & $+0.260^{c}$ & 26 & 7.78 & 8.10 \\
\hline $\mathrm{Ne}$ II $\lambda 4239.911$ & 34.632 & $-0.490^{\mathrm{c}}$ & & & \\
\hline Ne II $\lambda 4240.105$ & 34.632 & $-0.020^{\mathrm{c}}$ & 10 & 7.67 & 7.97 \\
\hline Ne II $\lambda 4250.645$ & 34.632 & $+0.150^{\mathrm{c}}$ & 25 & 8.04 & 8.36 \\
\hline $\mathrm{Ne}$ II $\lambda 4391.991$ & 34.737 & $+0.920^{\mathrm{c}}$ & 82 & 7.89 & 8.32 \\
\hline Mean... & $\cdots$ & $\cdots$ & $\cdots$ & $7.96 \pm 0.14$ & $8.43 \pm 0.19$ \\
\hline $\mathrm{Mg}_{\text {II }} \lambda 4481.126$ & 8.863 & +0.749 & & & \\
\hline $\mathrm{Mg}_{\text {II }} \lambda 4481.150$ & 8.863 & -0.553 & & & \\
\hline $\mathrm{Mg}_{\text {II }} \lambda 4481.325$ & 8.863 & +0.594 & Synth & 6.76 & 7.13 \\
\hline $\mathrm{A} 1 \mathrm{III} \lambda 3601.630$ & 14.376 & +0.01 & & & \\
\hline Al III $\lambda 3601.927$ & 14.374 & -0.95 & 100 & & 6.07 \\
\hline Al III $\lambda 4149.913$ & 20.55 & +0.63 & $\leqslant 8$ & & $\leqslant 5.06 ?$ \\
\hline Al III $\lambda 4479.885$ & 20.78 & +0.09 & & & \\
\hline Al III $\lambda 4479.971$ & 20.78 & +1.02 & & & \\
\hline Al III $\lambda 4480.009$ & 20.78 & -0.53 & 95 & & 6.22 \\
\hline $\mathrm{Al}$ III $\lambda 5722.730$ & 15.642 & -0.07 & 252 & & $7.14 ?$ \\
\hline Mean... & $\cdots$ & $\cdots$ & $\cdots$ & & $6.15 \pm 0.11$ \\
\hline Si III $\lambda 5739.734$ & 19.722 & -0.096 & 300 & 7.23 & 7.50 \\
\hline
\end{tabular}


Table 4

(Continued)

\begin{tabular}{|c|c|c|c|c|c|}
\hline \multirow[t]{2}{*}{ Line } & \multirow{2}{*}{$\begin{array}{c}\chi \chi \\
(\mathrm{eV})\end{array}$} & \multirow[b]{2}{*}{$\log g f$} & \multirow{2}{*}{$\begin{array}{c}W_{\lambda} \\
(\mathrm{m} \AA)\end{array}$} & \multicolumn{2}{|c|}{$\log \epsilon(\mathrm{X})$} \\
\hline & & & & NLTE $^{\mathrm{a}}$ & $\mathrm{LTE}^{\mathrm{b}}$ \\
\hline Si IV $\lambda 4116.104$ & 24.050 & -0.110 & 176 & 6.69 & 7.03 \\
\hline $\begin{array}{l}P_{\text {III }} \lambda 4080.089 \\
P_{\text {III }} \lambda 4222.198\end{array}$ & $\begin{array}{l}14.490 \\
14.610\end{array}$ & $\begin{array}{l}-0.310^{\mathrm{d}} \\
+0.210^{\mathrm{d}}\end{array}$ & $\begin{array}{r}20 \\
157\end{array}$ & & $\begin{array}{l}5.33 ? \\
5.91\end{array}$ \\
\hline Mean... & $\cdots$ & $\cdots$ & $\cdots$ & & $5.91 \pm 0.00$ \\
\hline P IV $\lambda 3347.736$ & 28.132 & $+0.25^{\mathrm{e}}$ & 19 & & 6.02 \\
\hline P IV $\lambda 3364.467$ & 28.132 & $+0.02^{\mathrm{e}}$ & 12 & & 6.01 \\
\hline P IV $\lambda 4249.656$ & 29.012 & $-0.13^{\mathrm{e}}$ & 11 & & 5.85 \\
\hline Mean... & $\cdots$ & $\ldots$ & $\cdots$ & & $5.96 \pm 0.10$ \\
\hline S III $\lambda 3324.854$ & 17.745 & +0.057 & 40 & 6.11 & 6.05 \\
\hline S III $\lambda 3656.560$ & 18.187 & -0.921 & 6 & 6.20 & 6.08 \\
\hline$S_{\text {III }} \lambda 3661.942$ & 18.192 & -0.462 & 15 & 6.12 & 6.06 \\
\hline S III $\lambda 3717.771$ & 18.244 & -0.060 & 65 & 6.29 & 6.32 \\
\hline S III $\lambda 3778.903$ & 18.193 & -1.148 & 7 & 6.39 & 6.33 \\
\hline Mean... & $\cdots$ & $\cdots$ & $\ldots$ & $6.22 \pm 0.12$ & $6.17 \pm 0.14$ \\
\hline Fe III $\lambda 3603.888$ & 11.210 & $-1.380^{c}$ & 13 & 6.00 & 6.61 \\
\hline Fe III $\lambda 4005.039$ & 11.570 & $-1.760^{\mathrm{c}}$ & $\leqslant 9$ & $\leqslant 6.10$ & $\leqslant 6.83$ \\
\hline Fe III $\lambda 4122.780$ & 20.599 & $+0.360^{\mathrm{c}}$ & $\leqslant 8$ & $\leqslant 6.40$ & $\leqslant 6.75$ \\
\hline Fe III $\lambda 4137.764$ & 20.613 & $+0.630^{\mathrm{c}}$ & $\leqslant 8$ & $\leqslant 6.00$ & $\leqslant 6.47$ \\
\hline Fe III $\lambda 4139.350$ & 20.613 & $+0.520^{\mathrm{c}}$ & $\leqslant 8$ & $\leqslant 6.20$ & $\leqslant 6.57$ \\
\hline Fe III $\lambda 4140.482$ & 20.613 & $+0.100^{\mathrm{c}}$ & $\leqslant 12$ & $\leqslant 6.80$ & $\leqslant 7.18$ \\
\hline Fe III $\lambda 4164.731$ & 20.634 & $+0.920^{\mathrm{c}}$ & $\leqslant 9$ & $\leqslant 5.90$ & $\leqslant 6.24$ \\
\hline Fe III $\lambda 4166.840$ & 20.634 & $+0.410^{\mathrm{c}}$ & $\leqslant 9$ & $\leqslant 6.40$ & $\leqslant 6.75$ \\
\hline Mean... & $\cdots$ & $\ldots$ & $\ldots$ & $6.00 \pm 0.00$ & $6.61 \pm 0.00$ \\
\hline
\end{tabular}

Notes.

a $\left(T_{\text {eff }}, \log g, \xi\right)=(25,000,2.50,24.0)$.

${ }^{\mathrm{b}}\left(T_{\text {eff }}, \log g, \xi\right)=(24,750,2.65,30.0)$.

${ }^{c}$ Kurucz $g f$-value.

${ }^{\mathrm{d}}$ Wiese et al. (1969).

${ }^{\mathrm{e}}$ Froese Fischer et al. (2006).

Si III. A search of the UVES spectra provided the wellresolved line at $5739.7 \AA$ (RMT 4) with no obvious blends-see Table 4 for the LTE and non-LTE Si abundances. Another line at $3791.4 \AA$ is blended with an O II line. Assuming that this line is solely due to $\mathrm{Si}$ III gives $\mathrm{Si}$ abundances of 7.03 and 7.14 for LTE and non-LTE analyses, respectively. These values are about 0.6 dex less than the abundances from the $5739 \AA$ line. Inclusion of the $\mathrm{O}$ II contribution to the $3791 \AA$ line obviously increases the abundance difference between the $5739 \AA$ and $3791 \AA$ lines.

Two additional multiplets are present on the CASPEC and UCLES spectra and provide six clean lines well suited to abundance analysis: three lines of RMT 2 at 4552.6, 4567.8, and $4574.7 \AA$ and three lines or blends of RMT 9 at 4829.1 , 4819.7, and 4813.3 $\AA$. (JH93 did not include these lines in their analysis.) These features give consistent abundances when analyzed with the appropriate model atmosphere: the mean nonLTE Si abundances are 6.86 (RMT 2) and 6.84 (RMT 9) for the CASPEC lines and 6.92 (RMT 2) and 6.75 (RMT 9) for the UCLES lines for the grand mean of 6.84 .

Si IV. The two Si IV lines of RMT 1 were detected and analyzed by JH93. The weaker line at $4116.104 \AA$ appears unblended. The stronger line at $4088.86 \AA$ is blended with an O II line from RMT 48 , as noted by Jeffery and Heber. These lines which appear also in the UCLES and UVES spectra are the only representatives of the $\mathrm{Si}^{3+}$ ion in the spectra.

Analysis of the $4116 \AA$ line on the UVES spectrum gives $\mathrm{Si}$ abundances of $\log \epsilon(\mathrm{Si})=7.03$ (LTE) and 6.69 (non-LTE).
A consideration of the Si II, Si III, and Si IV lines discussed in this section suggests that the $\mathrm{Si}$ abundance is $\log \epsilon(\mathrm{Si}) \simeq 6.8$ according to the suite of the CASPEC, UCLES, and UVES spectra. In drawing this conclusion, we give zero weight to the Si abundance from Si II $4128 \AA$ and $4130 \AA$ on the grounds that the $\mathrm{Si}$ II lines in the red spectrum are in emission, which feature is not accounted for by our non-LTE analysis. A concern is that the clean Si III $5739 \AA$ line gives a higher abundance of 7.2, and the blended Si III $3791 \AA$ line gives the upper limit of 6.6 when the line is assumed to be purely from the $\mathrm{Si}^{2+}$ ion.

Phosphorus. A search of UVES spectra was conducted for the P II, P III, and P IV lines. No lines of P II were found. Two lines of P III and three lines of P IV were deemed potentially suitable for abundance analysis. Three lines of P III were the basis for the $\mathrm{P}$ abundance reported by $\mathrm{JH} 93$.

$P$ III. Two lines appear to be unblended: $4080.09 \AA$ from RMT 1 and $4222.20 \AA$ from RMT 3. A second line from RMT 3 at the $4246.72 \AA$ line was listed by JH93, but this is blended with the $\mathrm{C}$ III and N II lines. The NIST Web site's $\log g f$-values are from the evaluation by Wiese et al. (1969). Recent quantum calculations by Froese Fischer et al. (2006) give slightly different results: the 2006 values are 0.17 dex and 0.08 dex smaller for RMT 1 and 3, respectively. We adopt the 2006 calculations. Abundances are LTE estimates because TLUSTY does not include model P ions.

In the UVES spectrum, the $4080 \AA$ line has a much smaller equivalent width than the $4222 \AA$ lines ( $20 \mathrm{~m} \AA$ versus $157 \mathrm{~m} \AA$ ) 
and yields a lower $P$ abundance (5.50 versus 5.99). The UCLES spectrum confirms this equivalent width difference. Yet the equivalent widths of the $4080 \AA$ and $4222 \AA$ lines listed by Jeffery and Heber from their CASPEC spectrum are almost identical. Examination of the CASPEC spectrum suggests that the $4080 \AA$ feature measured previously was displaced by about $0.5 \AA$ from the expected wavelength. In light of the agreement between the UVES and UCLES spectra, it would appear that the LTE analysis results in approximately a 0.5 dex abundance difference between the two lines. It does not appear that the $4222 \AA$ line is contaminated by an unidentified line or that the $4080 \AA$ is weakened by emission. Perhaps a non-LTE analysis would eliminate the $0.5 \mathrm{dex}$ difference.

$P$ IV. Lines of RMT 1 and 2 appear to be present as weak lines. the strongest two lines of RMT $1-3347.72 \AA$ and $3364.44 \AA$-are present, as is the single line of RMT 2 at $4249.57 \AA$. The latter line is too weak to be detected on either the CASPEC or UCLES spectra. The former two lines fall outside the CASPEC and UCLES bandpasses. The $\log g f$-values given by NIST are quantum calculations (Zare 1967). More recent calculations (Froese Fischer et al. 2006) giving similar results are adopted in Table 4.

With the exception of the low $\mathrm{P}$ abundance from the $\mathrm{P}$ III $4080 \AA$ line, a P LTE abundance of 6.0 is indicated from one $\mathrm{P}$ III and three $\mathrm{P}$ IV lines.

Sulfur. The S abundance given by JH93 was based on three S II and two S III lines. A reassessment of sulfur's contribution to DY Cen's spectrum was made using the UVES spectra. Atomic data were taken from Podobedova et al.'s (2009) critical evaluation.

$S$ II. Inspection of the UVES spectra gave no convincing identifications of S II lines. The likely strongest line- $5453.855 \AA$ from RMT 6-falls outside the bandpass of all available spectra. Several lines are not detectable on the UVES and/or the UCLES spectra and provide upper limits to the $S$ abundance consistent with the abundance provided by the $\mathrm{S}$ III lines. Three $\mathrm{S}$ II lines were measured by JH93: two lines (4815.6 $\AA$ and $4716.3 \AA$ ) from RMT 9 and one (4162.7 $\AA$ ) from RMT 44. On the UVES and UCLES spectra, the $4815.6 \AA$ line is absent, the $4716.3 \AA$ may be present, and the $4162.7 \AA$ line is blended with a C III line.

$S$ III. The ion $\mathrm{S}^{2+}$ is represented by a handful of weak lines. The two moderately strong lines from RMT 4 listed by Jeffery and Heber are badly blended. Table 4 details the selected lines providing abundance estimates; other lines from some of the multiplets are obviously present and might provide an abundance estimate were synthetic spectra computed. No line in Table 4 is of a strength to be detectable in the CASPEC spectrum.

The mean non-LTE sulfur abundance from the five $\mathrm{S}$ III lines is $\log \epsilon(\mathrm{S})=6.2$ after a negligible correction for non-LTE effects.

Argon. Leading lines of the lowest multiplets of Ar II are absent. In particular, $4400.986 \AA$ from RMT 1 with $\log g f=$ -0.28 is not detectable on the UVES spectra, and $4806.020 \AA$ with $\log g f=0.21$ is not present on the UCLES spectrum. The LTE Ar abundances are $\log \epsilon(\mathrm{Ar}) \leqslant 7.2$ and 6.6 from $4400 \AA$ and $4806 \AA$, respectively. Our equivalent width limit for $4806 \AA$ of $15 \mathrm{~m} \AA$ is similar to the measurement of $10 \mathrm{~m} \AA$ reported by JH93 from their CASPEC spectrum. All Ar I lines within the bandpasses of available spectra are far below detection limits.

Iron. JH93 used two Fe III lines from RMT 4 to derive the low $\mathrm{Fe}$ abundance $\log \epsilon(\mathrm{Fe})=5.04$, i.e., 2.4 dex below the solar abundance. Our search of the UVES spectrum yielded upper limits and one weak line from multiplets 4, 36, 45, and 118. The $\log g f$-values are taken from Kurucz.
Table 5

Summary of Photospheric Abundances

\begin{tabular}{lrrrr}
\hline \hline Element & Non-LTE & LTE & JH93 & Sun $^{\mathrm{a}}$ \\
\hline $\mathrm{H}$ & 10.7 & 10.8 & 10.8 & 12.0 \\
$\mathrm{He}$ & 11.5 & 11.5 & 11.5 & 10.9 \\
$\mathrm{C}$ & 9.6 & 9.8 & 9.5 & 8.4 \\
$\mathrm{~N}$ & 7.8 & 7.9 & 8.0 & 7.8 \\
$\mathrm{O}$ & 9.0 & 9.1 & 8.9 & 8.7 \\
$\mathrm{~F}$ & $\ldots$ & 7.1 & $\ldots$ & 4.6 \\
$\mathrm{Ne}$ I & 8.7 & 9.5 & 9.6 & 7.9 \\
$\mathrm{Ne}$ II & 8.0 & 8.4 & $\ldots$. & $\ldots$ \\
$\mathrm{Mg}$ & 6.7 & 7.1 & 7.3 & 7.6 \\
$\mathrm{Al}$ & $\ldots$ & 6.1 & 5.9 & 6.5 \\
$\mathrm{Si}$ & 6.8 & 7.0 & 8.1 & 7.5 \\
$\mathrm{P}$ & $\ldots$ & 6.0 & 5.8 & 5.4 \\
$\mathrm{~S}$ & 6.2 & 6.2 & 7.1 & 7.1 \\
$\mathrm{Ar}$ & $\ldots$ & $<6.9$ & 6.1 & 6.4 \\
$\mathrm{Fe}$ & 6.0 & 6.6 & 5.0 & 7.5 \\
\hline
\end{tabular}

Note. ${ }^{a}$ Asplund et al. (2009).

The leading line of RMT 4 at $4419.596 \AA$ is coincident with an emission line with emission of comparable strength present on the UCLES spectrum and just possibly on the CASPEC spectrum. Emission may be present also at $4431.019 \AA$ at a strength too weak to be seen in the UCLES spectrum: an absorption equivalent width is no stronger than $13 \mathrm{~m} \AA$ or the LTE abundance is less than $\log \epsilon(\mathrm{Fe})=7.0$. This is one of the two lines used by Jeffery and Heber, who gave the equivalent width as $67 \mathrm{~m} \AA$. Their other line at $4395.755 \AA$ is blended with an $\mathrm{O}$ II line. The RMT 4 line at $4352.577 \AA$ is absent, with an equivalent width limit of $6 \mathrm{~m} \AA$ yielding the non-LTE Fe abundance $\operatorname{limit} \log \epsilon(\mathrm{Fe}) \leqslant 7.2$.

Multiplet RMT 36 provides a possible detection of the multiplet's second strongest line: the line at $3603.888 \AA$ has an equivalent width of $13 \mathrm{~m} \AA$ and gives the non-LTE abundance of $\log \epsilon(\mathrm{Fe})=6.0$. Multiplets 45 and 118 provide useful upper limits to the Fe non-LTE abundance (see Table 4) in the range 6.0 to 6.4. In short, the adopted Fe non-LTE abundance is $\log \epsilon(\mathrm{Fe}) \simeq 6.0$.

A nagging concern about the abundance analysis is the 0.7 dex difference between the non-LTE Ne abundance provided by the red Ne I and blue-ultraviolet Ne II lines. The UVES spectra are the only available spectra of DY Cen to provide both Ne I and $\mathrm{Ne}$ II lines. The Ne I lines analyzed by JH93 confirm our Ne abundance from these lines. One might attribute the $0.7 \mathrm{dex}$ difference to an inadequate treatment of non-LTE effects in line formation in an atmosphere that fails to resemble the chosen theoretical atmosphere. One cannot help but notice that the redNe I-blue-Ne II abundance difference is reproduced apparently in abundances from the selection of Al III lines and Si III lines (see Table 4). Are these differences unrelated non-LTE effects? Or is there a common wavelength effect such as an error in the modeling of the continuous opacity?

\section{COMMENTARY ON DY CEN's COMPOSITION}

Table 5 summarizes the abundances. Columns two and three give our non-LTE and LTE abundances, respectively, and previous estimates by $\mathrm{JH} 93$ are given in column four. Composition of the solar photosphere is given in the final column (Asplund et al. 2009).

Comparison with Jeffery and Heber. A fair comparison involves the LTE abundance estimates. Inspection of Table 5 shows that the two determinations are in good agreement (i.e., 
differences of less than \pm 0.2 dex with the difference for $\mathrm{C}$ at 0.3 dex) except for three elements: $-1.1(\mathrm{Si}),-0.9(\mathrm{~S})$ and +1.6 $(\mathrm{Fe})$, where the numerical value is our LTE abundance minus their LTE abundance in dex. The principal reason for these differences appears to be found in differing choices of lines (see above).

Initial metallicity. DY Cen's initial metallicity should be judged from elements most likely unaffected by nucleosynthetic processes whose products are now in the star's atmosphere. This criterion identifies the sequence of heavy elements from $\mathrm{Mg}$ to Fe. For elements for which a non-LTE abundance was determinable, the differences (in dex) between abundance for DY Cen and the solar photosphere (Asplund et al. 2009) are -0.9 $(\mathrm{Mg}),-0.7(\mathrm{Si}),-0.9(\mathrm{~S})$, and $-1.5(\mathrm{Fe})$. A straight average of these four would give a metal deficiency of -1.0 dex. However, the $\mathrm{Mg} / \mathrm{Fe}, \mathrm{Si} / \mathrm{Fe}$, and $\mathrm{S} / \mathrm{Fe}$ ratios could point to the origin of DY Cen in the Galactic thick disk and an initial Fe abundance of about -1.2 dex.

For the remaining heavy elements with a LTE abundance estimate only, the differences with the solar photosphere are somewhat confusing for $\mathrm{Al}$ and $\mathrm{P}$ : $-0.3(\mathrm{Al})$ and $+0.6(\mathrm{P})$; however, as discussed above, the available lines for both elements do not provide an entirely consistent set of LTE abundances. Non-LTE corrections for Al seem likely to drive the $\mathrm{Al}$ abundance downward and provide a difference with the solar photosphere more in line with the values for $\mathrm{Mg}, \mathrm{Si}$, and $\mathrm{S}$. For an iron deficiency of 1.0 dex, the expected $\mathrm{P}$ abundance for the thick disk is about 4.7 (Caffau et al. 2011), i.e., a non-LTE correction of -1.3 dex is implied, or the chosen P III and P IV lines are seriously blended.

Fluorine. The fluorine abundance is in line with the abundance found for EHes by Pandey (2006) from F I lines and for many RCB stars also from F I lines by Pandey et al. (2008). The overabundance relative to the solar photosphere is 2.5 dex or 2.7 dex if the recent redetermination of the solar $\mathrm{F}$ abundance is adopted (Maiorca et al. 2014).

In the case of the EHes and RCrBs, synthesis of $\mathrm{F}$ is identified with a hot phase as two low-mass white dwarfs merge-the double-degenerate (DD) scenario. In the competing scenario for forming a $\mathrm{EHe}$ or a $\mathrm{RCrB}$, a low-mass asymptotic giant branch experiences a final or a late He shell flash, but F synthesis is not expected in this case. This latter expectation seems confirmed by the case of Sakurai's object (V4334 Sgr) for which Pandey et al. (2008) found an upper limit for the F abundance of 5.4 dex or about 1.6 dex less than the typical $\mathrm{F}$ abundance of EHes and most RCrBs.

Comparison with EHes. Spectral classification supported by the general characteristics of the chemical composition suggest that DY Cen's origin is closely related to that of the EHes whose origins in turn are supposed to be related to those of the RCB stars. Compilations of the compositions of EHes and RCBs (Pandey et al. 2006; Pandey \& Lambert 2011; Jeffery et al. 2011) show that key nucleosynthetic signatures of EHes are shared with DY Cen, i.e., DY Cen has the typical C/He ratio $(\sim 1 \%)$ and marked $\mathrm{Ne}$ and severe $\mathrm{F}$ overabundances of EHes. Similarity of nucleosynthetic signatures encourages the view that DY Cen and EHes share a common origin - a merger of a $\mathrm{He}$ and a $\mathrm{C}-\mathrm{O}$ white dwarf (i.e., the double-degenerate (DD) scenario). This view is, perhaps, challenged by the exceptional $\mathrm{H}$ abundance of DY Cen which is 2 dex greater than the next most H-rich EHe and less seriously tested by DY Cen's Fe abundance, which is among the lowest found for EHes. A severe challenge to the idea that DY Cen is a product of the DD scenario is the proposal by Rao et al. (2012) that the star is a spectroscopic binary with a low-mass unseen secondary which might be a He white dwarf or a low-mass (stripped?) main sequence star. Of course, one may suppose that DY Cen was originally a triple system which, thanks to a merger of two stars, is now a binary star.

Rao et al. (2012) suggest that DY Cen is a common-envelope system with the secondary presently embedded within the primary's envelope. Theoretical studies of the formation of H-poor stars in binaries (e.g., Podsiadlowski 2008) sketch how binary systems of two normal stars may evolve through a common envelope to either a single or binary H-poor compact star and then evolve to a sdB star, a hot compact H-poor star approaching a white-dwarf cooling track. Atomic diffusion alters the surface composition of a sdB star, and therefore it is difficult to correlate $\mathrm{sdB}$ compositions with the composition of putative predecessors. Common-envelope systems leading to a sdB in a binary (e.g., Podsiadlowski (2008), Figure 2), in contrast to merger scenarios, do not experience nucleosynthesis resulting in overabundances of $\mathrm{Ne}$ and, in particular, $\mathrm{F}$. Thus it would appear that a common envelope is insufficient to account for DY Cen, and a merger or similar event accompanied by nucleosynthesis is a necessary part of DY Cen's history. Nonetheless, it should be noted that the majority of sdB stars are binaries, and several have periods in the 10-100 d range (Maxted et al. 2001; Copperwheat et al. 2011; Barlow et al. 2012).

\section{CONCLUDING REMARKS}

Judged solely by effective temperature, surface gravity, and chemical composition, DY Cen is an EHe, albeit one with an unusually large amount of hydrogen. This identification of DY Cen as an EHe is in conflict with the identification of the star as a spectroscopic binary (Rao et al. 2012) and both the generally accepted idea that EHes form by the merger of two white dwarfs and the observational (if tentative) conclusion from radial velocity studies that (other) EHes are single stars. Resolution of the conflict is expected to come from an intensive spectroscopic campaign covering about $120 \mathrm{~d}$-three times the length of the orbital period found by Rao et al. Velocity and profile variations over this campaign should tease out the orbital velocity variation from those arising from atmospheric pulsations and wind instabilities.

We thank the referee Geoff Clayton for a constructive report. We would like to thank Anibal García-Hernández for his support; the UVES observations were obtained with his collaboration. G.P. and N.K.R. would like to thank Simon Jeffery for his hospitality at Armagh Observatory during 2013 January to February when a part of this work was done. We thank Vincent Woolf, who obtained and reduced the 2002 AAT/UCLES spectrum. D.L.L. acknowledges the support of the Robert A. Welch Foundation of Houston, Texas, through grant F-634.

\section{REFERENCES}

Asplund, M., Grevesse, N., Sauval, A. J., \& Scott, P. 2009, ARA\&A, 47, 481 Barlow, B. N., Wade, R. A., Liss, S. E., Østensen, R. H., \& Van Winckel, H. 2012, ApJ, 758, 58

Behara, N. T., \& Jeffery, C. S. 2006, A\&A, 451, 643

Bessell, M. S. 1990, PASP, 102, 1181

Caffau, E., Bonifacio, P., Faraggiana, R., \& Steffen, M. 2011, A\&A, 532, A98 Clayton, G. C., De Marco, O., Whitney, B. A., et al. 2011, AJ, 142, 54

Clayton, G. C., Geballe, T. R., Herwig, F., Fryer, C., \& Asplund, M. 2007, ApJ, 662,1220 
Copperwheat, C. M., Morales-Rueda, L., Marsh, T. R., Maxted, P. F. L., \& Heber, U. 2011, MNRAS, 415, 1381

De Marco, O., Clayton, G. C., Herwig, F., et al. 2002, AJ, 123, 3387

Dekker, H., D’Odorico, S., Kaufer, A., Delabre, B., \& Kotzlowski, H. 2000, Proc. SPIE, 4008, 534

Drilling, J. S. 1986, in IAU Colloq. 87: Hydrogen Deficient Stars and Related Objects, ed. K. Hunger, D. Schönberner, \& N. Kameswara Rao (Astrophysics and Space Science Library, Vol. 128; Dordrecht: Reidel), 9

Froese Fischer, C., Tachiev, G., \& Irimia, A. 2006, ADNDT, 92, 607

García-Hernández, D. A., Kameswara Rao, N., \& Lambert, D. L. 2011, ApJ, 739,37

Hoffleit, D. 1930, BHaro, 874, 13

Hubeny, I. 1988, CoPhC, 52, 103

Hubeny, I., \& Lanz, T. 1995, ApJ, 439, 875

Hubeny, I., Lanz, T., \& Jeffery, C. S. 1994, CCP7 Newsletter on Analysis of Astronomical Spectra (Daresbury, UK: Daresbury Laboratory), 30

Jeffery, C. S., \& Heber, U. 1993, A\&A, 270, 167

Jeffery, C. S., Karakas, A. I., \& Saio, H. 2011, MNRAS, 414, 3599

Kelleher, D. E., \& Podobedova, L. I. 2008a, JPCRD, 37, 709

Kelleher, D. E., \& Podobedova, L. I. 2008b, JPCRD, 37, 1285

Kelleher, D. E., \& Podobedova, L. I. 2008c, JPCRD, 37, 267

Kilkenny, D., \& Whittet, D. C. B. 1984, MNRAS, 208, 25

Maiorca, E., Uitenbroek, H., Uttenthaler, S., et al. 2014, ApJ, 788,149

Marino, B. F., \& Walker, W. S. G. 1971, VSSCi, 184, 7

Maxted, P. F. L., Heber, U., Marsh, T. R., \& North, R. C. 2001, MNRAS, 326,1391
Moore, C. E. 1972, A Multiplet Table of Astrophysical Interest (Washington, DC: National Bureau of Standards)

Moore, C. E. 1993, Tables of Spectra of Hydrogen, Carbon, Nitrogen, and Oxygen Atoms and Ions, ed. J. W. Gallagher, CRC Series in Evaluated Data in Atomic Physics (Boca Raton, FL: CRC Press)

Pandey, G. 2006, ApJL, 648, L143

Pandey, G., \& Lambert, D. L. 2011, ApJ, 727, 122

Pandey, G., Lambert, D. L., Jeffery, C. S., \& Rao, N. K. 2006, ApJ, 638, 454

Pandey, G., Lambert, D. L., \& Rao, N. K. 2008, ApJ, 674, 1068

Podobedova, L. I., Kelleher, D. E., \& Wiese, W. L. 2009, JPCRD, 38, 171

Podsiadlowski, P. 2008, in ASP Conf. Ser. 391, Hydrogen-Deficient Stars, ed. A. Werner \& T. Rauch (San Francisco, CA: ASP), 323

Pollacco, D. L., \& Hill, P. W. 1991, MNRAS, 248, 572

Przybilla, N., Butler, K., Heber, U., \& Jeffery, C. S. 2005, A\&A, 443, L25

Rao, N., Lambert, D. L., García-Hernández, D. A., \& Manchado, A. 2013, MNRAS, 431, 159

Rao, N. K., Giridhar, S., \& Lambert, D. L. 1993, A\&A, 280, 201

Rao, N. K., Lambert, D. L., García-Hernández, D. A., et al. 2012, ApJL, 760, L3

Saio, H., \& Jeffery, C. S. 2002, MNRAS, 333, 121

Schönberner, D. 1979, A\&A, 79, 108

Wiese, W., Smith, M., \& Glennon, B. M. 1966, NSRDS-NBS 4 (Washington, DC: National Bureau of Standards)

Wiese, W., Smith, M., \& Miles, B. 1969, NSRDS-NBS (Washington, DC: National Bureau of Standards)

Yan, C., Taylor, K., \& Seaton, M. 1987, JPhB, 20, 6399

Zare, R. N. 1967, JChPh, 47, 3561 